\title{
Accretion Disk Boundary Layers Around Neutron Stars: X-ray Production in Low-Mass X-ray Binaries
}

\author{
Robert Popham and Rashid Sunyaev ${ }^{1}$ \\ Max Planck Institut für Astrophysik \\ Karl-Schwarzschild-Strasse 1, 85740 Garching, Germany, 85740; \\ popham@mpa-garching.mpg.de, sunyaev@mpa-garching.mpg.de
}

\begin{abstract}
The boundary layer where the accretion disk meets the star is expected to be the dominant source of high-energy radiation in low-mass X-ray binaries which contain weakly magnetized accreting neutron stars. We present solutions for the structure of the boundary layer in such a system.

We find that the main portion of the boundary layer gas is hot $\left(\gtrsim 10^{8} \mathrm{~K}\right)$, has low density, and is radially and vertically extended. It will emit a large luminosity in X-rays, mainly produced by Comptonization of soft photons which pass through the hot gas. The gas is generally optically thick to scattering but optically thin to absorption. Energy is transported by viscosity from the rapidly rotating outer part of the boundary layer to the slowly rotating inner part, and this has the important effect of concentrating the energy dissipation in the dense, optically thick zone close to the stellar surface. Advection of energy also plays an important role in the energy balance. We explore the dependence of the boundary layer structure on the mass accretion rate and rotation rate of the star. We also examine the effects of changes in the $\alpha$ viscosity parameter and the viscosity prescription.

Radiation pressure is the dominant source of pressure in the boundary layer. The radiation flux in the boundary layer is a substantial fraction of the Eddington limiting flux even for luminosities well below ( $\sim 0.01$ times) the Eddington luminosity $L_{E d d}$ for spherically symmetric accretion. At luminosities near $L_{E d d}$, the boundary layer expands radially, and has a radial extent larger than one stellar radius. This radial expansion increases the surface area of the boundary layer and allows it to radiate a larger total luminosity.

Based on the temperatures and optical depths which characterize the boundary layer, we expect that Comptonization will produce a power-law
\end{abstract}

\footnotetext{
${ }^{1}$ also Space Research Institute, Moscow, Russia
} 
spectrum at low source luminosities. At high luminosities the scattering optical depth is quite large, and bremsstrahlung and Comptonization will produce a Planckian spectrum in the dense region where most of the energy is released. This spectrum will be altered by Comptonization as the radiation propagates through the lower-density outer boundary layer. We discuss some implications of our results for standard multi-component fits to X-ray spectra of LMXBs.

\section{Introduction}

\subsection{The Boundary Layer}

The boundary layer, the region where the rapidly spinning disk material reaches the more slowly spinning accreting star, is a crucial element of an accretion disk. In a thin accretion disk, the gas rotates at approximately the Keplerian velocity, and so by the time it approaches the surface of the accreting star, half of the gravitational potential energy released in the accretion process has been converted into rotational energy of the gas (in the Newtonian case). Unless the star is rotating rapidly, most of this energy will be released in the boundary layer. Furthermore, since this energy comes from a small region close to the star, the boundary layer should be hotter than the disk and should produce harder radiation. Thus, the boundary layer is expected to be the dominant source of high-energy emission from accretion disks, which for accretion onto neutron stars takes the form of X-rays.

The boundary layer region is quite complex, since the accreting gas must make the transition from a disk to a star, with the accompanying changes in the balance of momentum and energy. For example, as the rotation rate of the gas drops below Keplerian, rotational support against gravity is replaced by pressure support. The radiation which cools the disk flows vertically, from the disk midplane to the surface, while near the stellar surface radiation must flow radially outward. In general, the radial scale over which the disk properties vary becomes comparable to or smaller than the vertical scale of the disk. As

a result, radial transport, particularly of energy, plays an important role in the boundary layer.

Boundary layers around neutron stars are even more complex, due to a number of additional physical processes which become important due to the small size of the neutron star and the resulting strong gravity and enormous luminosity due to accretion. In the solutions we present here, radiation pressure plays a major role in the dynamics of the flow, and can increase the sound speed to $\gtrsim 0.1 c$. Radiation pressure is dominant even for 
accretion rates where the total luminosity is well below (0.01 times) the Eddington limit, since the local radiation flux still reaches a large fraction of the local Eddington value. The gas can reach very high temperatures, so Comptonization of soft incident photons can be an important energy loss mechanism and can produce power-law spectra (Sunyaev \& Titarchuk 1980). Energy transfer between protons and electrons can become inefficient at high temperatures, producing a two-temperature plasma. Relativistic effects can also be important, since the neutron star radius is comparable to the radius of the last stable particle orbit in the Schwarzschild metric. The incident radiation could in principle remove angular momentum from the gas (Miller \& Lamb 1993, 1996), and if the neutron star radius is smaller than the last stable orbit, the gas may spiral toward the surface (Kluźniak \& Wilson 1991).

\subsection{Observational Background}

Low-mass X-ray binaries (LMXBs) have been observed in X-rays for almost 40 years, since the discovery of Sco X-1 (Giacconi et al. 1962). Since then, around 100 other LMXBs have been discovered, and many of these have been observed extensively in X-rays and at other wavelengths. These observations have revealed much about the timing and spectral behavior of LMXBs.

The best-studied LMXBs generally fall into two classes, the atoll sources and the Z sources, based on the paths they trace out in the X-ray color-color diagram as they vary in brightness (Hasinger \& van der Klis 1989). These variations are believed to be due to changes in the mass accretion rate $\dot{M}$, with the $\mathrm{Z}$ sources having luminosities near the Eddington limit and the atoll sources varying over a wider range, down to about $1 \%$ of the Eddington limit. If so, then the atoll sources in particular provide an excellent means for directly observing the effects of changing $\dot{M}$ and checking the predictions of our models.

As the sources move along the paths in the color-color diagram, their variability properties also change, as indicated by changes in the shape of their power density spectra. In some regimes, quasi-periodic oscillations (QPOs) appear. Some of these are at relatively low frequencies of a few $\mathrm{Hz}$ or tens of $\mathrm{Hz}$ (van der Klis et al. 1985; Middleditch \& Priedhorsky 1986), but others are the recently discovered kHz QPOs (see van der Klis 1998 for a review). Sunyaev \& Revnivtsev (2000) have recently shown that power density spectra of LMXBs in the low/hard state show much more power at high frequencies $(i, 100 \mathrm{~Hz})$ than those of black hole candidates, probably due to the presence of the neutron star surface and the associated energy release in a boundary layer. The high-frequency variability and oscillations presumably originate from the innermost portions of the accretion flow, very 
near the neutron star, and provide strong motivation for studying these inner regions in detail.

Nonetheless, our understanding of the production of X-rays and the formation of the $\mathrm{X}$-ray spectrum in these sources is still very incomplete. The X-ray spectra of LMXBs are generally modeled in a rather simple way, by combining two or more spectral components to fit the overall spectrum. The components consist of blackbody, "disk" (a sum of blackbodies corresponding to annuli of a disk with a certain temperature and emissivity profile), bremsstrahlung, or power-law spectra. Thompson (elastic) scattering or Comptonization (where the frequency changes due to scattering) can play important roles in producing or modifying these components.

These model fits are useful in that they have provided a simple picture of the changes in LMXB spectra as a function of luminosity. At high luminosities, above around $10^{37} \mathrm{erg} \mathrm{s}^{-1}$, the data are fit well by blackbody-type spectra. Mitsuda et al. (1984) used a two-component model with a disk component and a single-temperature blackbody to fit high-luminosity LMXB spectra. White et al. (1986) used a similar model in which the disk emission was Compton-scattered to higher energies. At lower luminosities, the spectra are fit well by a power-law spectrum with an exponential cutoff at high energies (White, Stella, \& Parmar 1988). Both a soft component and a hard power law can be present at low luminosities, but the power law seems to disappear at higher luminosities (Barret \& Vedrenne 1994).

The components used in these fits incorporate much of our current knowledge of the processes by which hot gas emits X-rays. However, in order to better understand which components should be present and how their temperatures and luminosities relate to each other, it is important to have a model for the accretion flow near the neutron star. In particular, the boundary layer region, where the accretion disk meets the star, is expected to produce a large portion of the X-ray luminosity. The size, temperature, and optical depth of this region depend on the dynamics and energetics of the accretion flow near the stellar surface. In this paper, we study the boundary layer region in detail, as a step toward the eventual goal of being able to directly interpret LMXB spectra in terms of fundamental parameters such as the mass accretion rate and the rotation rate of the accreting neutron star.

\subsection{Theoretical Background}

The structure of the boundary layer region has been studied in other types of accreting systems, most notably cataclysmic variables (Pringle 1977; Pringle \& Savonije 1979; 
Tylenda 1981; Patterson \& Raymond 1985; Kley 1991; Narayan \& Popham 1993; Popham \& Narayan 1995) and pre-main sequence stars such as T Tauri and FU Orionis stars (Popham et al. 1993, 1996). Cataclysmic variables (CVs) are similar to LMXBs in many respects; the main difference is simply that accretion is onto a white dwarf instead of a neutron star. CVs also emit X-rays which are believed to originate in the boundary layer. Narayan \& Popham (1993, hereafter NP93) showed that the optical depth of the boundary layer region is sensitive to the mass accretion rate. At high accretion rates, the boundary layer is optically thick and emits approximately as a blackbody with an effective temperature of a few $\times 10^{5}$. But at low accretion rates, the boundary layer becomes optically thin to absorption and is unable to cool efficiently, as had been predicted by Tylenda (1981) and King \& Shaviv (1984). The accreting gas is heated to $\sim 10^{8} \mathrm{~K}$ by the energy dissipated in the boundary layer, and emits hard X-rays.

While we expect some similarities between boundary layers in CVs and in LMXBs, we also expect a number of major differences. Because a neutron star is so much smaller than a white dwarf, a much larger luminosity must be emitted from a much smaller area, resulting in much higher radiation fluxes and temperatures, and therefore Comptonization and radiation pressure play critical roles.

Studies of the accretion flow onto neutron stars have largely focused on the case where the neutron star has a very strong magnetic field. This field is believed to truncate the accretion disk at some inner radius and channel the accretion onto magnetic field lines, so that ultimately it falls onto polar caps corresponding to the poles of the magnetic field (Pringle \& Rees 1972; Basko \& Sunyaev 1976; Ghosh, Lamb, \& Pethick 1977). Evidence for this magnetically-channeled polar accretion is provided by the X-ray pulsations and magnetic cyclotron features first observed in Her X-1 (Trümper et al. 1978) and in a number of other X-ray pulsars. However, the great majority of LMXBs do not show any evidence for periodic pulsations or cyclotron features. In these systems the magnetic field may be sufficiently small ( $B \lesssim 10^{8} \mathrm{G}$, as in some millisecond pulsars) to allow the disk to extend all the way in to the stellar surface, resulting in a boundary layer region where the rapidly rotating disk meets the (presumably) more slowly rotating star.

There have only been a few studies of the inner accretion flow onto non-magnetic neutron stars (those where the stellar magnetic field is not strong enough to alter the flow), but for the most part they have not computed the boundary layer structure in detail. Sunyaev \& Shakura (1986) computed the relative contributions of the disk and boundary layer to the total accretion luminosity. In standard Newtonian disk theory each contribute half of the total, but in the Schwarzschild metric the boundary layer contributes more than the disk. The relative contributions depend on the neutron star radius $R_{*}$ : if $R_{*}$ equals the 
radius $R_{m s}=6 G M / c^{2}$ of the marginally stable particle orbit, the boundary layer luminosity should be about twice that of the disk. If $R_{*}<R_{m s}$ and the accreting gas spirals rapidly in from $R_{m s}$ to $R_{*}$, the relative contribution of the boundary layer is even larger. The relative luminosities of the disk and boundary layer also depend on the rotation rate of the star, as shown by Sibgatullin \& Sunyaev (1998), who included the effects of rotation on the shape of the star and its gravitational field. Shakura \& Sunyaev (1988) derived analytic estimates for the boundary layer structure at low accretion rates and X-ray luminosities $L_{x}<10^{36} \mathrm{erg} \mathrm{s}^{-1}$, assuming constant temperature and viscosity coefficient. They did not address the case of higher luminosities, where radiation pressure should dominate. Kluźniak \& Wilson (1991) computed the structure of an accretion belt on the stellar surface under the assumption that $R_{*}<R_{m s}$, and the accreting gas impacts the surface at high velocity, and found that high temperatures and hard spectra would be produced. King \& Lasota (1987) argued that the boundary layer region would reach high temperatures even if the gas does not experience rapid infall, because as in the CV case, the gas cannot cool efficiently enough to radiate away the dissipated energy. They show that for luminosities less than $\sim 10^{35} \mathrm{erg} \mathrm{s}^{-1}$, where gas pressure is dominant, the boundary layer region should heat up and expand vertically to form a "corona" around the neutron star.

Recently, Inogamov \& Sunyaev (1999) have studied the problem of disk accretion onto neutron stars, using a new approach for modeling the boundary layer region. The accreting gas arrives at the equator of the star spinning at the Keplerian velocity, and forms a layer on the stellar surface which spreads from the equator toward the poles. As the gas moves meridionally, it loses angular momentum and dissipates energy, which is radiated away from the surface. This approach essentially treats the boundary layer as part of the star rather than part of the disk, and the angular velocity decreases with latitude on the stellar surface rather than with radial distance from the surface. This complements the approach used in the current paper, and later we compare the results of the two approaches.

We model the boundary layer as part of the disk, using the slim disk equations (Paczyński \& Bisnovatyi-Kogan 1981; Muchotrzeb \& Paczyński 1982; Abramowicz et al. 1988), which contain terms which allow for large deviations from the standard thin Keplerian disk with efficient cooling. A similar approach has been used in most previous studies of boundary layers in CVs and accreting pre-main sequence stars. This approach has the advantage that it allows one to solve for the structure of the disk and boundary layer together, using a single set of equations throughout. The interface with the accreting star is treated as a set of boundary conditions implemented at the stellar radius. We use Newtonian equations throughout, despite the fact that our adopted neutron star radius of $10 \mathrm{~km}$ is less than the radius of the marginally stable particle orbit $(12.4 \mathrm{~km}$ for a neutron star mass of $1.4 \mathrm{M}_{\odot}$ ). Since there have been no previous solutions of these equations for 
neutron star parameters, either in Newtonian or relativistic form, we feel that a Newtonian solution is an important first step. The effects of relativity will be added in the future.

Figure 1 shows some of the important features of our results. At the transition from the disk to the boundary layer, the angular velocity $\Omega$ reaches a maximum and the flow passes through a narrow neck, where the disk height is only $\sim 40-70$ meters in the low- $\dot{M}$ solutions. The radial extent of the boundary layer region is $\sim 0.1-0.2$ of the stellar radius at low $\dot{M}$, and the height is comparable to the radial extent. The angular velocity drops slowly over most of the boundary layer, and then rapidly at the inner edge. At high $\dot{M}$ near the Eddington limit, the situation is quite different: the radial extent and height boundary layer are equal to the stellar radius, and the neck between the disk and boundary layer is

much wider. Here the drop in angular velocity occurs over the whole width of the layer.

In $\S 2$, we describe the slim disk equations and radiative transfer scheme which we have used to model boundary layers in LMXBs. We present expressions for the viscous transport of energy in the disk and boundary layer. We present the results of our calculations in $\S 3$, and show how the transport of energy by viscosity, radiation, and advection play essential roles in determining the boundary layer structure. We present solutions for a variety of mass accretion rates, stellar rotation rates, and viscosities, and show how the size, temperature, and other properties vary. In $\S 4$ we discuss the energetics of the boundary layer, the behavior near the Eddington limit, and the implications of our results for the spectra of LMXBs.

\section{Boundary Layer Model}

\subsection{Dynamics}

We use the slim disk equations to describe accretion in the disk and boundary layer. Like the standard thin disk equations, these describe the disk structure as a function of radius. In the vertical direction, we assume simple approximate relations. The slim disk equations are a generalization of the standard thin disk equations which include terms that become important when the disk deviates from a thin, Keplerian configuration. These additional terms often involve radial derivatives. The equations are solved assuming a steady state, and the solutions extend from the radius of the stellar surface (assumed to be $10 \mathrm{~km}$ ) out to 100 times the stellar radius. Boundary conditions for the flow are set at both the inner and outer radii, and the equations are solved using a relaxation method.

The slim disk equations have been presented in a number of previous papers, and therefore we only give a brief description of them here; for more details consult, e.g. 
Popham \& Narayan (1995, hereafter PN95). The mass accretion rate through the disk, which is constant with radius under the steady-state assumption, is given by

$$
\dot{M}=-4 \pi R H \rho v_{R},
$$

where $\dot{M}$ is the mass accretion rate, $R$ is the radius, $H$ is the disk vertical scale height, $\rho$ is the mass density, and $v_{R}$ is the radial velocity.

The material in the disk rotates with angular velocity $\Omega$. Viscosity transfers angular momentum down the gradient of $\Omega$, i.e. from an annulus with higher $\Omega$ to an adjacent annulus with lower $\Omega$. In a steady-state disk, the flow of angular momentum is given by

$$
\dot{M} \frac{d}{d R}\left(\Omega R^{2}\right)=\frac{d}{d R}\left(4 \pi R^{2} H w_{r \phi}\right),
$$

where $w_{r \phi}=\rho \nu R d \Omega / d R$ is the viscous stress, and $\nu$ is the viscosity coefficient. This can be integrated to obtain

$$
\dot{M} \frac{\nu}{v_{R}} \frac{d \Omega}{d R} R^{2}=\dot{M} \Omega R^{2}-\dot{J} .
$$

The integration constant $\dot{J}$ is the angular momentum accretion rate. We expect that there will be a maximum in $\Omega$ at a point close to the surface of the star where the Keplerian disk ends and the boundary layer begins, and $\dot{J}$ must be equal to $\dot{M} \Omega R^{2}$ at this point since $d \Omega / d R=0$. Therefore we write

$$
\dot{J} \equiv j \dot{M} \Omega_{K}\left(R_{*}\right) R_{*}^{2}
$$

where $R_{*}$ is the radius of the neutron star, and $\Omega_{K}\left(R_{*}\right) \equiv\left(G M / R_{*}^{3}\right)^{1 / 2}$ is the Keplerian angular velocity at $R$. The parameter $j$ is the ratio of $\dot{J}$ to the usual value $\dot{M} \Omega_{K}\left(R_{*}\right) R_{*}^{2}$ assumed in the thin disk equations, where the radial extent of the boundary layer is assumed to be very small. Since we wish to obtain the thickness of the boundary layer from our solutions, we allow $j \neq 1$.

The radial momentum equation is

$$
v_{R} \frac{d v_{R}}{d R}+\frac{1}{\rho} \frac{d P}{d R}-\frac{1}{\rho} \frac{d}{d R}\left(\rho \nu \frac{d v_{R}}{d R}\right)=\left(\Omega^{2}-\Omega_{K}^{2}\right) R,
$$

where $P$ is the total pressure. The terms on the left-hand side of this equation represent the radial acceleration of the accreting gas, radial pressure gradient, and the radial viscous acceleration, all of which can be important in the boundary layer.

The standard energy equation, which assumes that the ions and electrons have the same temperature, is

$$
\rho H v_{R} T_{c} \frac{d S}{d R}+\frac{1}{R} \frac{d}{d R}\left(R H F_{R}\right)=\rho H \nu\left(R \frac{d \Omega}{d R}\right)^{2}-F_{V},
$$


where $T_{c}$ is the temperature at the disk midplane, $S$ is the entropy, and $F_{R}$ and $F_{V}$ are the radial and vertical radiative fluxes, respectively. The terms on the right-hand side of this equation are the viscous dissipation in the disk and the radiation from the disk surface; these are assumed to be equal at all radii in the standard thin disk equations. The terms on the left-hand side represent radial transport of energy and are not included in the thin disk equations. The first is the entropy advected inward with the accreting gas; note that in an advection-dominated disk this term approximately balances the viscous dissipation. The second is transport by radial radiation flux, which is generally quite important in disk boundary layers.

The energy equation given above applies when the energy transfer from ions to electrons via Coulomb collisions proceeds on a shorter timescale than the other heating and cooling processes. This may not be true in the boundary layer region, where the gas may become hot and rarefied. Therefore we use separate energy equations for the ions and the electrons. The ions are heated by the energy dissipation in the disk and cooled by Coulomb collisions with the electrons

$$
\rho H v_{R} T_{i} \frac{d S_{i}}{d R}=\rho H \nu\left(R \frac{d \Omega}{d R}\right)^{2}-Q_{p e} H
$$

where $Q_{p e}=(3 / 2) n_{p} k\left(T_{i}-T_{e}\right) / \tau_{p e}$ is the Coulomb cooling rate, and $\tau_{p e}=2.11 \times 10^{-23} T_{e}^{3 / 2} / \rho$ s (Spitzer 1962) is the Coulomb energy transfer timescale, with $\rho$ in $\operatorname{cgs}$ units and $T_{e}$ in degrees Kelvin. The ion temperature $T_{i}$ may be substantially higher than the electron temperature $T_{e}$. The electrons are heated by the Coulomb collisions, and the electron energy equation also includes the radiation

$$
\rho H v_{R} T_{e} \frac{d S_{e}}{d R}+\frac{1}{R} \frac{d}{d R}\left(R H F_{R}\right)=Q_{p e} H-F_{V} .
$$

The total pressure is the sum of the gas and radiation pressures

$$
P=\mathcal{R} \rho\left(T_{i}+T_{e}\right)+\frac{4 \pi}{3 c} u_{c}
$$

where we have assumed the gas is ionized hydrogen, $\mathcal{R}$ is the gas constant, and $u$ is the mean radiative intensity as defined below. The entropy advection terms for the electrons and ions are given by

$$
\begin{array}{r}
T_{e} d S_{e}=\mathcal{R} T_{e}\left(\frac{3}{2} d \ln T_{e}-d \ln \rho\right)+\frac{4 \pi}{c \rho}\left(d u_{c}-\frac{4}{3} u_{c} d \ln \rho\right), \\
T_{i} d S_{i}=\mathcal{R} T_{i}\left(\frac{3}{2} d \ln T_{i}-d \ln \rho\right),
\end{array}
$$


respectively, where we have included the radiation terms in the equation for the electrons.

We estimate the vertical pressure scale height of the disk in the usual way. The vertical pressure gradient must balance the vertical component of the gravity of the star

$$
-\frac{1}{\rho} \frac{d P}{d z}=\frac{G M}{R^{2}} \frac{z}{R}=z \Omega_{K}^{2}(R)
$$

Taking $P=\rho c_{s}^{2}$, i.e., using $c_{s}=(P / \rho)^{1 / 2}$ to define the approximate sound speed, and assuming that the disk is isothermal so that $c_{s}(z)$ is constant, we find $\rho(z)=\rho(0) \exp \left(-\Omega_{K}^{2} z^{2} / 2 c_{s}^{2}\right)$. Therefore we define the vertical pressure scale height as $H=\sqrt{2} c_{s} / \Omega_{K}$. Alternatively, if we assume that the pressure is dominated by radiation, we have $-(1 / \rho) d P / d z=\kappa F_{V} / c=\Omega_{K}^{2}(R) z$, where $\kappa$ is the opacity and $F_{V}$ the vertical radiation flux. As discussed later, we assume that the vertical flux increases linearly with $z, F_{V}(z)=F^{\prime} z$, with $F^{\prime}$ constant. This gives a quadratic variation for the pressure: $P(z)=P(0)-\rho \kappa F^{\prime} z^{2} / 2 c$, with $P$ reaching zero at $z=\left(2 P(0) c / \rho \kappa F^{\prime}\right)^{1 / 2}=\sqrt{2} c_{s} / \Omega_{K}$, which is what we have defined as $H$.

\subsection{Viscosity and Turbulence in the Boundary Layer}

The origin and nature of the viscosity in the boundary layer may be very different from that in the Keplerian disk. The viscosity may arise from turbulence, but the origin of this turbulence is not known. There are several well-known theoretical and experimental results on the generation of turbulence which may be relevant to the conditions within the boundary layer. Two of the best known mechanisms should not operate in the boundary layer:

1) The boundary layer is stable against linear hydrodynamic instabilities according to the Rayleigh criterion, since the specific angular momentum of the matter increases strongly with radius within the boundary layer, $d\left(\Omega R^{2}\right) / d R>0$ (Rayleigh 1916).

2) The leading candidate for producing the viscosity in the disk is the magnetorotational instability originally discovered by Velikhov (1959) and Chandrasekhar (1960) and applied to accretion disks by Balbus and Hawley (1997 and references therein). However, this instability arises when $d \Omega^{2} / d R<0$, which is true in the Keplerian disk, but not true in the boundary layer.

Nonetheless, there are other ways in which turbulence within the boundary layer might be generated. Two of these, which are probably closely related, are:

1) Experimental studies of flow between two rotating cylinders have shown that 
turbulence can arise for high Reynolds numbers in the case where the outer cylinder rotates much faster than the inner one (see, e.g., Schlichting 1957; Joseph 1976 and references therein). This case is not relevant to the main Keplerian portion of the disk, but might be relevant to the boundary layer. [ Therefore we need to estimate the Reynolds number Re in the boundary layer. For the boundary layer around an accreting neutron star, the radiative viscosity $\nu_{\text {rad }}=(4 / 15)\left(U_{\text {rad }} / \kappa_{s} \rho^{2} c\right)$ (Weinberg 1972), where $U_{\text {rad }}$ is the radiative energy density, should be well in excess of the molecular viscosity, as discussed by Inogamov \& Sunyaev (1999), and in the absence of turbulence the radiative viscosity would be dominant. We can calculate a posteriori the Reynolds number $R e=\Omega R\left(R-R_{*}\right) / \nu_{\text {rad }}$ in our boundary layer solutions based on the radiative viscosity. We find rather low values $R e \sim 10^{2}$ over much of the boundary layer, but much larger values $R e \sim 10^{6}-10^{11}$ near the inner and outer edges (Fig. 2).

2) If the radial extent of the boundary layer is small compared to the radius, $\Delta R \ll R$, then we can neglect the curvature in the first approximation, and consider the flow to be similar to flow near a wall. In this situation, we know from experiments that turbulence may arise at high Reynolds numbers. For $\dot{M}=10^{-9} \mathrm{M}_{\odot} \mathrm{yr}^{-1}$, the radial extent of the boundary layer is $\Delta R \ll R$, and this approximation may be valid; however, for $\dot{M}=10^{-8} \mathrm{M}_{\odot} \mathrm{yr}^{-1}$ we have $\Delta R \sim R$, and we cannot neglect the curvature of the flow. This case is probably very similar to the previous one, but there is much more experimental evidence available.

Since we are treating the boundary layer as the inner part of the accretion disk and using disk equations to describe it, we have chosen the simple approach of applying the same viscosity law in the disk and the boundary layer. We use an $\alpha$ viscosity (Shakura \& Sunyaev 1973); however, in defining the viscosity coefficient, we also use the radial pressure scale height $H_{r}=P /|d P / d R|$ (Papaloizou \& Stanley 1986). The viscosity coefficient is then defined as $\nu=\alpha c_{s} l_{\text {turb }}$, where $l_{\text {turb }}=\left(H^{-2}+H_{r}^{-2}\right)^{-1 / 2}$; this essentially takes the turbulent length scale $l_{\text {turb }}$ to be the smaller of the two scale heights. We do not include any reduction in the viscosity due to causality (Narayan 1992; Narayan, Loeb \& Kumar 1994); however, we find that the radial velocity stays well below the sound speed in our solutions, so that causal corrections are unimportant.

Another limit on the viscosity is the criterion that the length scale for the turbulence should be short enough that the change in azimuthal velocity $\Delta v_{\phi}$ over that length should be less than the sound speed (Shakura \& Sunyaev 1988). One can thus define a third length

\footnotetext{
${ }^{2} \mathrm{~A}$ possible exception to this would be the case where the neutron star is rotating rapidly; the fastest measured rotation frequencies of millisecond pulsars and X-ray bursters are around $600 \mathrm{~Hz}$, corresponding to angular velocities of $\sim 1 / 3$ of $\Omega_{K}\left(R_{*}\right)$.
} 
scale $H_{s} \equiv c_{s} /\left|d v_{\phi} / d R\right|$ which should limit the turbulence. Since we want the viscosity to be limited by the smallest of the three scales $H, H_{r}$, and $H_{s}$, we can adopt a similar

prescription to the one above, where $l_{\text {turb }}=\left(H^{-2}+H_{r}^{-2}+H_{s}^{-2}\right)^{-1 / 2}$. We will refer to this prescription as "subsonic" viscosity.

At the mass accretion rates and luminosities we consider, the gas is radiation pressure dominated. Since our sound speed is based on the total pressure including both gas and radiation pressure, it is important that the gas and the radiation are coupled. As long as the optical depth across the turbulent length scale is $z 1$, the gas and radiation should be reasonably well coupled. Since the turbulent length scale is $\sim H$, and since scattering dominates the opacity, this amounts to the condition that the disk be optically thick to scattering. As we will see later, all of our solutions satisfy this criterion, although at the lowest mass accretion rates which we consider, the optical depth of the boundary layer from the midplane to the surface is only a few. If the gas density were to drop too low, so that the disk became optically thin, the radiation would stream freely through it, and we would need to treat the turbulence in a more detailed way.

\subsection{Radiative Transfer}

We also need to compute the radiative fluxes in the vertical and radial directions, $F_{V}$ and $F_{R}$, which appear in the energy equation. The radiative transfer in the boundary layer region can be rather complicated due to the rapid variations in the gas temperature and density. In the case of LMXBs the situation is further complicated by Compton scattering when the gas reaches high temperatures.

We use a simple scheme for describing the radiative transfer in the boundary layer region, in the spirit of the dynamical equations described above. In particular, we make simplifying assumptions about the vertical dependence of the variables, and ignore the frequency dependence of the radiation and opacity. In most respects, our equations are the same as those used by PN95 and described in detail in Appendix A of that paper. However, we have modified the transfer equations to include an approximate treatment of Compton scattering.

We use a "four-stream" treatment. The eight intensities corresponding to the directions of the corners of a cube reduce to four because we assume axisymmetry: $I^{++}, I^{+-}, I^{-+}, I^{--}$, where the superscripts refer to the radial and vertical directions, respectively. We define four moments of these intensities:

$$
u=\frac{1}{4}\left(I^{++}+I^{+-}+I^{-+}+I^{--}\right)
$$




$$
\begin{aligned}
v_{x} & =\frac{1}{4 \sqrt{3}}\left(I^{++}+I^{+-}-I^{-+}-I^{--}\right) \\
v_{z} & =\frac{1}{4 \sqrt{3}}\left(I^{++}-I^{+-}+I^{-+}-I^{--}\right) \\
w & =\frac{1}{4}\left(I^{++}-I^{+-}-I^{-+}+I^{--}\right)
\end{aligned}
$$

which correspond to the mean intensity, the radial and vertical fluxes, and a cross term, respectively.

In place of the usual transfer equation for each of the intensities, we use a modified version with a Compton energy amplification term included, e.g.,

$$
\frac{1}{\sqrt{3}} \frac{d I^{++}}{d x}+\frac{1}{\sqrt{3}} \frac{d I^{++}}{d z}=-\rho \kappa I^{++}+\rho \kappa_{s} u(1+\delta)+\rho \kappa_{a} B,
$$

where $\kappa, \kappa_{s}, \kappa_{a}$ are the total, scattering, and absorptive opacities, $\delta$ is the energy amplification factor due to Compton scattering, and $B=\sigma T_{e}^{4} / \pi$ is the mean intensity of blackbody radiation at the electron temperature $T_{e}$. The amplification factor is written as $\delta=\left(T_{e}-T_{\text {phot }}\right) / T_{\text {com }}$, where $T_{\text {com }}=m_{e} c^{2} / 4 k \simeq 1.5 \times 10^{9} \mathrm{~K}$. The "photon temperature" $T_{\text {phot }}=T_{e} \bar{\epsilon} / \bar{\epsilon}_{B B}$, where $\bar{\epsilon}$ is the mean photon energy and $\bar{\epsilon}_{B B} \simeq 2.7 k T_{e}$ is the mean photon energy of a blackbody distribution. We define $\bar{\epsilon}=u / N$, where $N$ is the mean photon number intensity (photons $\mathrm{cm}^{-2} \mathrm{sr}^{-1} \mathrm{~s}^{-1}$ ). We calculate $N$ using a set of equations similar to those above, but with number intensities instead of energy intensities, the blackbody mean number intensity $N_{B}=B / \bar{\epsilon}_{B B}$ taking the place of $B$, and no Compton enhancement factor $\delta$, since Compton scattering conserves photon number.

When we combine the transfer equations for the four directions, we obtain four differential equations

$$
\begin{aligned}
\frac{\partial v_{x}}{\partial x}+\frac{\partial v_{z}}{\partial z} & =\rho \kappa_{a}(B-u)+\rho \kappa_{s} u \delta \\
\frac{\partial u}{\partial x}+\frac{\partial w}{\partial z} & =-3 \rho \kappa v_{x} \\
\frac{\partial w}{\partial x}+\frac{\partial u}{\partial z} & =-3 \rho \kappa v_{z} \\
\frac{\partial v_{z}}{\partial x}+\frac{\partial v_{x}}{\partial z} & =-\rho \kappa w .
\end{aligned}
$$

These are identical to the equations without a Compton factor (eqs. A2.9-A2.12 of PN95), except for the second term on the right-hand side of the first equation, which gives the Compton scattering contribution to the total flux divergence. 
We apply boundary conditions and assume vertical dependences as in PN95; namely, $v_{z}$ and $w$ are zero at the disk midplane $(z=0)$ and increase linearly with $|z|$ at rates $v_{z}^{\prime}$ and $w^{\prime}$, and $u$ and $v_{x}$ have their maximum values $u_{c}, v_{x c}$ at the midplane, and decrease quadratically with $|z|$. At the surface $(z=H)$ the incoming intensities $I^{+-}, I^{--}$are assumed to be zero, so $u=\sqrt{3} v_{z}$ and $w=\sqrt{3} v_{x}$. We also convert the $x$-derivatives to $R$-derivatives, and find

$$
\begin{aligned}
\frac{1}{R H} \frac{\partial\left(R H v_{x c}\right)}{\partial R}+v_{z}^{\prime} & =\rho \kappa_{a}(B-u)+\rho \kappa_{s} u \delta \\
\frac{\partial u}{\partial x}+w^{\prime} & =-3 \rho \kappa v_{x} \\
\frac{\partial w^{\prime}}{\partial R}+\frac{3 \tau+2 \sqrt{3}}{H} v_{z}^{\prime} & =\frac{2 u_{c}}{H^{2}} \\
\frac{\partial v_{z}^{\prime}}{\partial R}+\frac{\tau+2 / \sqrt{3}}{H} w^{\prime} & =\frac{2 v_{x c}}{H^{2}}
\end{aligned}
$$

where $\tau=\kappa \rho H$ is the vertical optical depth.

\subsection{Viscous Energy Transport}

A very important term in the energy balance of accretion disks is the energy transport due to viscosity. This energy transport is an unavoidable consequence of the viscous angular momentum transport that makes accretion possible.

\subsubsection{In the Boundary Layer}

The local rate of viscous dissipation of kinetic energy into thermal energy per unit surface area of the disk is given by

$$
Q^{+}=w_{r \phi} H R \frac{d \Omega}{d R}=\rho \nu H R^{2}\left(\frac{d \Omega}{d R}\right)^{2}
$$

(Shakura \& Sunyaev 1973). This expression is a specific case of the general formula for viscous energy dissipation (Landau \& Lifshitz 1959). Using the continuity and angular momentum equations (1) and (3), we can write this in the form

$$
Q^{+}=-\frac{\dot{M}}{4 \pi R}\left(\Omega R^{2}-j \Omega_{K}\left(R_{*}\right) R_{*}^{2}\right) \frac{d \Omega}{d R} .
$$

The ultimate source of the dissipated energy is the gravitational potential energy 
released as the gas falls in toward the star

$$
Q_{\text {grav }}=\frac{\dot{M}}{4 \pi R} \frac{d}{d R}\left(\frac{-G M}{R}\right)=\frac{\dot{M}}{4 \pi R} \frac{G M}{R^{2}}=\frac{\dot{M}}{4 \pi R} \Omega_{K}^{2} R .
$$

However, if the radial extent of the boundary layer is very small, $\Delta R \ll R_{*}$, the gravitational energy release within the boundary layer is only a small fraction $\Delta R / R$ of the total accretion luminosity. Practically all of the energy dissipated in the boundary layer then comes from the kinetic energy of the gas, which is lost as the rotational velocity decreases from Keplerian to zero (for a non-rotating star). The kinetic energy is lost at a rate

$$
Q_{k i n}=\frac{1}{4 \pi R} \frac{d}{d R}\left(\frac{1}{2} \dot{M} \Omega^{2} R^{2}\right) \simeq \frac{\dot{M}}{4 \pi R}\left(\Omega R^{2} \frac{d \Omega}{d R}\right),
$$

per unit disk surface area, where the last expression assumes that the boundary layer is narrow so $\Omega$ varies much more rapidly than $R$.

If the boundary layer is wide, with $\Delta R \sim R_{*}$, then gravitational energy release within the boundary layer becomes important. Also, our expression for the rate of kinetic energy loss must include a term proportional to $\Omega^{2} R$ to account for the change in radius. The resulting total gravitational and kinetic energy loss rate per unit disk surface area is

$$
Q_{\text {grav }}+Q_{\text {kin }}=\frac{\dot{M}}{4 \pi R}\left(\Omega_{K}^{2} R+\Omega R^{2} \frac{d \Omega}{d R}+\Omega^{2} R\right)
$$

Both this and the expression in Eq. 20 differ from the local dissipation rate given in Eq. 18.

The reason for this is the viscous transport of energy, which redistributes energy within the boundary layer. Viscosity causes both angular momentum and energy to be carried down the local gradient in $\Omega$, i.e. from regions of higher $\Omega$ to regions of lower $\Omega$. In the Keplerian disk, this means that angular momentum and energy are carried outward. At the dividing line between the disk and the boundary layer, $\Omega$ reaches its maximum value and $d \Omega / d R=0$. In the boundary layer, $\Omega$ decreases inward, so viscosity carries both angular momentum and energy inward toward the stellar surface.

The energy transported by viscosity is given by $\Omega N$, where $N$ is the torque

$$
\Omega N=-4 \pi R^{2} H w_{r \phi} \Omega=-4 \pi R H \rho \nu R^{2} \frac{d \Omega}{d R} \Omega=\dot{M} \Omega \frac{\nu R^{2}}{v_{R}} \frac{d \Omega}{d R} .
$$

Note that this expression is proportional to $d \Omega / d R$; as mentioned above, energy is carried down the angular velocity gradient together with the angular momentum. Also, energy is not carried across a maximum in $\Omega$, such as the boundary between the disk and boundary layer. Therefore viscous transport does not transfer energy between the disk and boundary 
layer, but redistributes it within the disk and the boundary layer. We can rewrite the viscous energy transport rate using Eq. 3 as

$$
\Omega N=\dot{M}\left(\Omega^{2} R^{2}-j \Omega \Omega_{K}\left(R_{*}\right) R_{*}^{2}\right) .
$$

The energy per unit disk surface area deposited at a given radius is given by the divergence of the viscous energy transport rate

$$
Q_{\text {trans }}=-\frac{1}{4 \pi R} \frac{d}{d R}(\Omega N)=-\frac{\dot{M}}{4 \pi R} \frac{d}{d R}\left(\Omega^{2} R^{2}-j \Omega \Omega_{K}\left(R_{*}\right) R_{*}^{2}\right) .
$$

This energy is the main reason for the difference between the local viscous dissipation rate (Eq. 18) and the local gravitational and kinetic energy release (Eq. 20 or 21). Other terms - the energy associated with the change in pressure, the bulk viscous dissipation, and the kinetic energy of radial motion (i.e., terms proportional to those on the left-hand side of Eq. 3) - can also play a role; we have omitted these here for simplicity.

In the boundary layer, $\Omega$ decreases inward, and so the viscous transport removes energy from the outer boundary layer and carries it inward toward the star. The gradient $d \Omega / d R$ is much larger, by a factor $\gtrsim R_{*} / \Delta R$, in the boundary layer than in the disk. In addition, if $\Omega$ does not decrease uniformly through the whole boundary layer, but instead drops more rapidly in some sections than in others, viscous transport will be even stronger in those sections. Overall, we expect that viscous transport should be very important in determining the energy balance and overall structure of the boundary layer. This expectation is verified by the solutions presented in $\S 3$.

\subsubsection{In the Disk}

The expressions given above apply to the Keplerian disk as well. In a Keplerian disk, half of the gravitational energy released goes into the rotational kinetic energy of gas. This kinetic energy is released later in the boundary layer.

As in the boundary layer, the local energy dissipation in the disk does not match the local rate of gravitational and kinetic energy release. For example, taking $\Omega=\Omega_{K}$ in Eq. 18 gives $Q^{+}=(3 / 8 \pi) \dot{M} \Omega_{K}^{2}\left[1-j\left(R_{*} / R\right)^{1 / 2}\right]$, while the total gravitational and kinetic energy loss rate per unit disk surface area is is $(1 / 8 \pi) \dot{M} \Omega_{K}^{2}$. This has the well-known result that at radii $R \gg R_{*}$, the dissipation rate is 3 times larger than expected (see Shakura \& Sunyaev 1973).

If we take $\Omega=\Omega_{K}$ in Eq. 24 above, we find that the energy per unit disk surface area deposited by viscous transport is $Q_{\text {trans }}=(1 / 4 \pi)\left[1-(3 / 2) j\left(R_{*} / R\right)^{1 / 2}\right] \dot{M} \Omega_{K}^{2}$. This is 
exactly the difference between the actual dissipation rate and the expected dissipation rate based on the change in gravitational potential and kinetic energy. The energy deposited by viscous transport is positive for $R>(9 / 4) j^{2} R_{*}$ and negative for smaller radii; energy is removed from the inner region of the disk, and the dissipation rate there is much lower than the rate of gravitational potential and kinetic energy loss. Note, however, that none of this energy comes from the boundary layer, since viscous transport cannot carry energy across the maximum in $\Omega$.

\subsubsection{Importance for the Spectrum}

In $\S 3$ we calculate the rate of viscous transport in our boundary layer solutions, and find that it plays an important role in determining the boundary layer structure. Most importantly, the viscous transport causes most of the kinetic energy of the gas to be dissipated in the densest part of the boundary layer. There the gas is rotating slowly and the radiation flux is well below Eddington, so centrifugal and radiation forces do not support the gas against gravity. The density increases rapidly as the gas piles up on the stellar surface. This is crucial because it will make the radiation spectrum quite different from that which would be produced if all the energy were dissipated in the low-density regions farther outside.

\section{Results}

We begin by showing a typical solution with an intermediate mass accretion rate $\dot{M}=10^{-9} \mathrm{M}_{\odot} \mathrm{yr}^{-1}$, the viscosity parameter $\alpha=0.1$, and the stellar rotation rate $\Omega_{*}=0$. These three quantities characterize our solutions. We use a neutron star mass of $1.4 \mathrm{M}_{\odot}$ and a neutron star radius of $10^{6} \mathrm{~cm}$ for all of our calculations. Thus the accretion luminosity is for this solution is $L_{a c c}=G M \dot{M} / R_{*}=1.17 \times 10^{37} \mathrm{erg} \mathrm{s}^{-1}$. Some equations of state for neutron stars predict a slightly larger radius, and if the neutron star is rotating, the star will be flattened and the equatorial radius will increase.

In discussing this solution, we begin with the disk and follow the flow of the accreting gas inward through the boundary layer and onto the star. In general, the disk portion of our solutions closely resembles the standard thin disk solutions (Shakura \& Sunyaev 1973). The additional "slim disk" terms in the equations are small.

The boundary layer region can be seen clearly in Fig. 3. As the gas flows in, $\Omega$ begins to deviate from Keplerian at $R \simeq 1.19 \times 10^{6} \mathrm{~cm}$. In fact, one can see in the inset that 
the rotation first becomes super-Keplerian[ at $R \simeq 1.19 \times 10^{6} \mathrm{~cm}$, then drops back below Keplerian at $R \simeq 1.17 \times 10^{6} \mathrm{~cm}$. The pressure drops as the gas moves from the disk into the boundary layer (Fig. 4a), and the resulting inward pressure gradient produces the small super-Keplerian zone.

At this radius, the gas infall accelerates rapidly; $v_{R}$ increases by more than two orders of magnitude (Fig. 3), so the surface density of the disk drops accordingly. Note that the sound speed $c_{s}$ is shown as a dashed line in Fig. 3 ; in the hot boundary layer region, the radial Mach number $v_{R} / c_{s}$ is larger than in the disk, which indicates that advection of energy becomes important there. Nonetheless, $v_{R} \ll c_{s}$ in all of our solutions, so a causal viscosity prescription would make little difference.

The sudden drop in surface density produces a fairly abrupt transition in the vertical optical depth of the accreting gas. The disk is quite optically thick to both absorption and scattering, while the boundary layer is still optically thick to scattering but optically thin to absorption, even when the effects of scattering are included. The scattering optical depth also reaches a minimum of $\tau_{s} \sim 4$ in the boundary layer, measured from the midplane to the surface. The radial optical depth through the boundary layer is of the same order as the vertical optical depth.

When the infalling gas becomes optically thin to absorption, the gas temperature rises dramatically as radiative cooling becomes inefficient (Fig. 3). (Note that the electron temperature shown in the plots is always the midplane temperature; the electron temperature near the disk surface may be quite a bit lower, depending on the optical depth of the gas, and on the vertical dependence of the energy dissipation.) The effective temperature also peaks in this region at $T_{\text {eff }} \sim 2 \times 10^{7} \mathrm{~K}$; this is simply a measure of the flux from the disk surface $F_{V}=\sigma T_{e f f}^{4}$ and is not indicative of the spectrum produced by the boundary layer gas.

The high temperature and radiation pressure causes the gas to expand vertically, further lowering the density and the absorptive opacity. The vertical expansion is quite dramatic, with the vertical scale height going from less than $1 \%$ of the stellar radius in the innermost part of the disk to $\sim 25 \%$ of the stellar radius in the boundary layer (Fig. 3 ).

\footnotetext{
${ }^{3}$ This small super-Keplerian region seems to be present in most of our solutions, and is due to the drop in pressure as the gas moves from the disk into the boundary layer. The exceptions are solutions with high values of $\dot{M}$, where the pressure increases from the disk to the boundary layer, and those in which the star is spinning at nearly breakup speed, so that no boundary layer is present. It has been argued that such a region is a necessary condition for the onset of an advection-dominated region in a disk (Abramowicz, Igumenshchev, \& Lasota 1998).
} 
Nonetheless, the implied vertical velocity of the gas $v_{z} \simeq v_{R} d H / d R<c_{s}$, so the gas stays in hydrostatic equilibrium.

As the gas moves inward through the hot boundary layer, $\Omega$ drops rather gradually, and the pressure increases again. Note that almost all of the pressure in the hot region is due to radiation, while in the innermost disk gas pressure dominates (Fig. 4b). The outward pressure gradient provides support against gravity, compensating for the sub-Keplerian rotation (Fig. 5). Bulk viscous effects also play a small but non-negligible role. Note that the glitches in the pressure gradient and bulk viscous terms at $R \simeq 1.038 \times 10^{6} \mathrm{~cm}$ in Fig. 5 are a numerical problem associated with a change in grid resolution at that point. At $R \simeq 1.04 \times 10^{6} \mathrm{~cm}, \Omega$ is around $75-80 \%$ of the Keplerian value, so about $60 \%$ of the support against gravity comes from rotation.

Here the gas begins to pile up on the surface of the star, with $v_{R}$ decreasing quite rapidly (Fig. 3). The gas makes a transition back to an optically thick state, and the temperature drops rather abruptly. $\Omega$ drops quite rapidly to the stellar rotation rate. The height of the disk also drops abruptly, so that the disk is again rather thin as it reaches the stellar surface. This seems somewhat counterintuitive, and may be a consequence of using simple one-dimensional disk equations to approximate a fundamentally two-dimensional flow. One might expect the gas to stay at a large height above the midplane and fall onto the stellar surface in a wide belt, rather than falling back toward the midplane. Even if this is the case, we expect the qualitative picture presented here to be correct, since whether the local vertical scale height is $5 \%$ or $50 \%$ of $R_{*}$, the gas must slow down and get much denser when it reaches the stellar surface.

The innermost zone of the solution, inside $R \simeq 1.035 \times 10^{6} \mathrm{~cm}$, serves as the "star" in our calculation. This portion of the solution is calculated using the same disk equations which are used everywhere else. The gas settles slowly inward, maintaining the same $\dot{M}$ as in the disk and boundary layer, with very low radial velocity and very high density. The energy balance is between inward advection of the gas entropy and outward radial radiation flux. The gas is quite hot and the vertical (i.e. meridional) pressure scale height is generally around $5-10 \%$ of $R$. Gas pressure again dominates over radiation pressure here; this is difficult to see in Fig. 4a due to the steep increase in both the total pressure and the radiation pressure, but it can be seen clearly in Fig. 4b. The viscosity given by the $\alpha$ prescription is very high and so $\Omega$ is nearly constant at the value set by the boundary conditions, $\Omega \simeq \Omega_{*}$.

The mean intensity of radiation $u$ is several orders of magnitude smaller than the blackbody mean intensity $B$ (calculated from the electron temperature) in the hot, low-density region of the boundary layer (Fig. 4c). The temperature (and thus $B$ ) is 
high within this low optical depth zone; however, $u$ decreases monotonically with $R$. The photon and electron temperatures diverge in the same region (Fig. $4 \mathrm{~d}$ ). In the outer part of the boundary layer, the photon temperature exceeds the electron temperature and Comptonization heats the electrons (cf. Eq. 14 and the following discussion). Inside $R \simeq 1.08 \times 10^{6} \mathrm{~cm}$, the electron temperature exceeds the photon temperature, so the photons cool the electrons due to Comptonization. Note that the ion temperature also diverges from the electron temperature in the boundary layer region, reaching $\sim 10^{9} \mathrm{~K}$.

The radiation flux is predominantly radial in the inner part of the boundary layer (Fig. 4 e) - this radiation carries away the energy dissipated in the region where $\Omega$ drops rapidly. This radiation is scattered by the hot boundary layer gas and escapes from its surface. We have used the angle $\tan ^{-1}\left(F_{R} / F_{V}\right)$ to indicate the general direction of the radiation (Fig. 4f). It should be noted that $F_{R}$ is the radial flux at the disk midplane where $F_{V}$ is zero by symmetry, while $F_{V}$ is the vertical flux at the disk surface where $F_{R}$ is generally small. (Since we do not explicitly solve the vertical structure of the disk, $F_{V}$ and $F_{R}$ are assumed to linearly increase and quadratically decrease with distance from the midplane, respectively; see PN95 for details.) Thus the ratio $F_{R} / F_{V}$ does not give the true direction of the flux at any real location in the disk, but we have used it as a convenient way to show how the average direction of the flux changes with radius.

In the outer part of the boundary layer, the dominant terms in the energy equation are Compton scattering and energy advection (Fig. 5). Note that the energy terms in this figure are vertically integrated so that they are in units of energy flux per unit midplane area of the disk. In the outer boundary layer, the gas is heated by scattering of energetic photons from farther in. Inside $R \simeq 1.08 \times 10^{6} \mathrm{~cm}$, the situation reverses; here the photons are cooling the hot gas. Thus Comptonization has the net effect of carrying energy outward in the hot region, while advection carries energy inward. Throughout this low-density region, the viscous dissipation is rather small compared to the Compton and advection terms, and the emitted radiation (the $\rho \kappa_{a}(B-u)$ term in Eq. 16a) is negligible. This changes when the gas density increases near the stellar surface (Fig. 5). Here the viscous dissipation is large, and the Compton scattering deposits some energy, but dwindles as the electron and photon temperatures reach equilibrium. The denser gas radiates the dissipated energy away efficiently; most of the emitted photons travel radially outward into the hot, low-density region and are inverse Compton scattered to higher energies. 


\subsection{Viscous Energy Transport}

The strong peak in the viscous dissipation at the inner edge of the boundary layer comes from two sources. The first is the kinetic energy lost by the gas as $\Omega$ drops rapidly. However, an even larger source is the energy transported radially by viscosity.

Figure 5 shows the viscous transport $\Omega N=\dot{M}\left(\Omega^{2} R^{2}-\Omega j \Omega_{K}\left(R_{*}\right) R_{*}^{2}\right)$, which carries energy outward in the disk and inward in the boundary layer. Note that the viscous transport is zero at $R \simeq 1.16 \times 10^{6} \mathrm{~cm}$, where $\Omega$ reaches its maximum value. The local rate of energy deposition by viscous transport depends on the radial gradient of the transport rate $-d(\Omega N) / d R=-\dot{M} d\left(\Omega^{2} R^{2}-\Omega j \Omega_{K}\left(R_{*}\right) R_{*}^{2}\right) / d R$. Where $\Omega N$ decreases with radius, as in the inner boundary layer from $R \simeq 1.035-1.0365 \times 10^{6} \mathrm{~cm}$, or in the disk outside $R \simeq 2.5 \times 10^{6} \mathrm{~cm}$, viscous transport deposits energy. Between $R \simeq 1.0365 \times 10^{6} \mathrm{~cm}$ and $R \simeq 2.5 \times 10^{6} \mathrm{~cm}, \Omega N$ increases with radius, so viscous transport removes energy.

The rate of energy gain or loss due to viscous transport is shown in the bottom panels of Fig. 5, together with the local viscous dissipation rate and the rate of loss of kinetic energy, all in units of the local gravitational energy release per unit radius $\left(G M \dot{M} / R^{2}\right)$. The most dramatic result is that the viscous transport is almost fully responsible for producing the very sharp peak in the dissipation rate in the dense inner boundary layer. The kinetic energy loss is also quite concentrated, reaching more than 100 times the local gravitational release, but the viscous transport deposits energy at a rate exceeding 600 times the local gravitational release. The middle panel shows that over the entire outer boundary layer, the gravitational potential and kinetic energy lost by the gas is not dissipated locally, but instead is transported inward and dissipated in a very narrow layer near the surface of the star, where the gas density is much higher than in the outer boundary layer.

The peak dissipation occurs in a zone where the radial optical depth for the emitted photons to reach the hot, low-density outer boundary layer is about 20, as shown in Fig. 6 . The vertical optical depth at this radius is much larger than 20, so most of the radiation travels radially outward. Note that in this figure it appears that viscous transport deposits much more energy in the dense region than it takes away from the low-density region, but this is simply a consequence of the fact that we have plotted the energy deposition as a function of the radial optical depth. In fact, the viscous transport term can only redistribute the energy within the boundary layer, and as Fig. 5 shows, the region from which viscous transport removes energy is far larger than the region where it deposits energy. The fact that the energy is dissipated in the dense, optically thick gas, rather than in the rarefied gas of the outer boundary layer, makes a major difference in the radiation spectrum of the boundary layer. 
Far from the star, Fig. 5 shows that the energy deposited per unit disk surface area by viscous transport of energy $(1 / 4 \pi)\left[1-(3 / 2) j R_{*}^{1 / 2} / R^{1 / 2}\right] \dot{M} \Omega_{K}^{2}$ increases the viscous dissipation rate to $Q^{+}=(3 / 8 \pi)\left(1-j R_{*}^{1 / 2} / R^{1 / 2}\right) \dot{M} \Omega_{K}^{2}$. At very large radii, this is three times larger than the $(1 / 8 \pi) \dot{M} \Omega_{K}^{2}$ expected from the change in the gravitational potential energy and kinetic energy of the gas. The energy deposited by viscous transport is positive for $R>(9 / 4) j^{2} R_{*}$; in the solution shown in Fig. $5, j \simeq 1.064$, so the viscous transport deposits energy for $R \gtrsim 2.55 R_{*}$ and removes energy for $R \lesssim 2.55 R_{*}$.

\subsection{Mass Accretion Rate}

Figure 7 shows the effects of varying $\dot{M}$. The solutions cover the range $\dot{M}=10^{-8}-10^{-10} \mathrm{M}_{\odot} \mathrm{yr}^{-1}$; the corresponding accretion luminosities are $L_{a c c}=G M \dot{M} / R_{*}=1.17 \times 10^{37}\left(\dot{M} / 10^{-9} \mathrm{M}_{\odot} \mathrm{yr}^{-1}\right) \mathrm{erg} \mathrm{s}^{-1}$, part of which should be emitted by the boundary layer and part by the disk. The most apparent effect of increasing $\dot{M}$ is the dramatic radial expansion of the boundary layer region. The location of the outer edge of the boundary layer is clear in the figures; $\Omega$ deviates from $\Omega_{K}$ and decreases as the gas falls inward, while all the other variables show a sudden increase (in temperature, radial velocity and disk scale height) or decrease (in density and optical depth). At $10^{-10} \mathrm{M}_{\odot} \mathrm{yr}^{-1}$ the radial extent of the region is only about $10 \%$ of the stellar radius, whereas at $10^{-8} \mathrm{M}_{\odot} \mathrm{yr}^{-1}$ it extends to $R \simeq 2 R_{*}$. The dramatic increase in the boundary layer width for high $\dot{M}$ occurs because $10^{-8} \mathrm{M}_{\odot} \mathrm{yr}^{-1}$ is very close to the Eddington limit, as discussed further below.

The profile of $\Omega$ in the boundary layer also changes as a function of $\dot{M}$. As discussed above, $\Omega$ drops more gradually in the region where the density is lower, and then quite rapidly in the denser region near the stellar surface. As $\dot{M}$ increases, more of the drop in $\Omega$ occurs in the low-density region: at $\dot{M}=10^{-10} \mathrm{M}_{\odot} \mathrm{yr}^{-1}$, more than $90 \%$ of the drop in $\Omega$ is in the dense region, while at $10^{-8} \mathrm{M}_{\odot} \mathrm{yr}^{-1}$ less than $20 \%$ is in the dense region. This, together with the changing width of the low-density region, means that the energy dissipation is concentrated in a far smaller volume at low than at high $\dot{M}$.

The optical depth of the boundary layer increases steadily with increasing $\dot{M}$. It is marginally optically thick to scattering at $10^{-10} \mathrm{M}_{\odot} \mathrm{yr}^{-1}$, but $\tau_{s} \gtrsim 100$ for all radii at $10^{-8} \mathrm{M}_{\odot} \mathrm{yr}^{-1}$. Nonetheless, the free-free opacity is so low that the boundary layer remains optically thin to absorption at all accretion rates, even when the effects of multiple scattering are taken into account. Even at $10^{-8} \mathrm{M}_{\odot} \mathrm{yr}^{-1}$, the effective optical depth $\tau_{*}=\left(\tau_{s} \tau_{a}\right)^{1 / 2} \simeq 0.001$ 
The temperature profile of the boundary layer changes substantially as a function of $\dot{M}$. Solutions for all values of $\dot{M}$ show a jump in $T$ in the low-density region, and over most of the region, the higher values of $\dot{M}$ produce higher temperatures, ranging from $\sim 1.5-5.5 \times 10^{8} \mathrm{~K}$ as $\dot{M}$ increases. However, the lower values of $\dot{M}$ produce double-peaked temperature profiles, and the inner peak reaches higher temperatures as $\dot{M}$ decreases, reaching $T \sim 8 \times 10^{8} \mathrm{~K}$ at $\dot{M}=10^{-10} \mathrm{M}_{\odot} \mathrm{yr}^{-1}$. The difference between the ion and electron temperatures is also sensitive to $\dot{M}$; at $10^{-10} \mathrm{M}_{\odot} \mathrm{yr}^{-1}, T_{i, \max } \sim 2 \times 10^{10} \mathrm{~K} \sim 25 T_{e, \text { max }}$, while at $10^{-8} \mathrm{M}_{\odot} \mathrm{yr}^{-1}$, the Coulomb coupling is much more efficient and there is practically no difference between $T_{i}$ and $T_{e}$.

\subsubsection{The Ratio of Disk and Boundary Layer Luminosities}

One important effect of the radial expansion of the boundary layer is to change the boundary layer and disk luminosities. As we discussed in $\S 2.3$, viscous energy transport cannot carry energy across the maximum in $\Omega$ which occurs at the outer edge of the boundary layer. Thus, the luminosity of the disk must equal the change in the gravitational potential and kinetic energy of the gas within the disk. If the boundary layer is very small, then the disk and boundary layer luminosities are about the same. Half of the total accretion luminosity is released in the disk, and the other half goes into rotational kinetic energy, which is released in the boundary layer. As the transition between the disk and boundary layer moves to radii significantly larger than $R_{*}$, the amount of energy released in the disk decreases, and that energy is radiated by the boundary layer instead. For instance, if the transition is at $R=2 R_{*}$, only $1 / 4$ of the total accretion luminosity is released in the disk, and $3 / 4$ in the boundary layer (1/4 from the kinetic energy of the gas at $R=2 R_{*}$, and $1 / 2$ from the gravitational energy released as the gas moves from $2 R_{*}$ to $R_{*}$ ). This would make the ratio of boundary layer to disk luminosity 3:1 instead of the usual 1:1 (for the Newtonian case; in the Schwarzschild metric the ratio is about 2:1 (Sunyaev \& Shakura 1986)). This could have important effects on the overall radiation spectrum.

\subsubsection{Effect of Flux from the Neutron Star}

Note that we have assumed in our calculations that the radiative flux across $R_{*}$ is very small. The accreted hydrogen should burn on the surface of the star and release a luminosity $\sim 0.007 \dot{M} c^{2} \simeq 4 \times 10^{35}\left(\dot{M} / 10^{-9} \mathrm{M}_{\odot} \mathrm{yr}^{-1}\right) \mathrm{erg} \mathrm{s}^{-1}$. If radiated evenly from the whole surface of the star, this produces a flux $\sim 3.2 \times 10^{22}\left(\dot{M} / 10^{-9} \mathrm{M}_{\odot} \mathrm{yr}^{-1}\right) \mathrm{erg} \mathrm{cm}^{-2} \mathrm{~s}^{-1}$. We have carried out calculations in which the radiative flux at the inner boundary is set 
to this value. The resulting solutions are almost identical to those with no flux at the boundary. The reason for this is clear from Fig. 8; the radial flux at the inner edge of the boundary layer reaches $\sim 0.1-1.4 \times 10^{25} \mathrm{erg} \mathrm{cm}^{-2} \mathrm{~s}^{-1}$ for $\dot{M}=10^{-10}-10^{-8} \mathrm{M}_{\odot} \mathrm{yr}^{-1}$, respectively, a significant fraction of the Eddington flux. The flux from nuclear burning on the stellar surface would have to reach a significant fraction of this value before it had a major effect on the boundary layer structure. Such fluxes are probably reached during X-ray bursts.

\subsection{Accretion Rates Near the Eddington Limit}

The radial extent of the boundary layer becomes much larger at $\dot{M}=10^{-8} \mathrm{M}_{\odot} \mathrm{yr}^{-1}$. This accretion rate is close to the Eddington limiting rate, which for a spherical geometry is $\dot{M}_{E d d}=4 \pi c R_{*} / \kappa_{s} \simeq 1.73 \times 10^{-8} \mathrm{M}_{\odot} \mathrm{yr}^{-1}$ for $R_{*}=10^{6} \mathrm{~cm}$. In a disk geometry, the crucial quantities are the radiation flux in the radial direction $F_{R}$ and the Eddington limiting flux $F_{E d d}=\left(G M / R^{2}\right) c / \kappa_{s}$; these are shown in Fig. 8. The radiation provides an increasing fraction of the support against gravity as the gas moves inward through the boundary layer. This is reflected in the gradual decrease of the radial velocity $v_{R}$ as the gas flows through the boundary layer in the $10^{-8} \mathrm{M}_{\odot} \mathrm{yr}^{-1}$ solution. Note that the radial velocity profile is rather different from the solutions for lower values of $\dot{M}$ which are not so close to the Eddington limit (Fig. 7).

Even though the radial flux in the $\dot{M}=10^{-8} \mathrm{M}_{\odot} \mathrm{yr}^{-1}$ solution is very close to the local Eddington value, we are able to find solutions for larger values of $\dot{M}$. These solutions are shown in Fig. 9. The radial expansion of the boundary layer is dramatic: at $\dot{M}=10^{-7.85} \mathrm{M}_{\odot} \mathrm{yr}^{-1} \simeq 1.4 \times 10^{-8} \mathrm{M}_{\odot} \mathrm{yr}^{-1}$, the outer edge of the boundary layer is at $R \simeq 3 \times 10^{6} \mathrm{~cm}$. Thus the radial extent of the boundary layer doubles for a $40 \%$ increase in $\dot{M}$. Note that $1.4 \times 10^{-8} \mathrm{M}_{\odot} \mathrm{yr}^{-1}$ is still less than the Eddington limit for spherical accretion, and of course part of the luminosity is released in the disk, so the luminosity released in the boundary layer is well below the spherical Eddington limit. On the other hand, the disk covers only a fraction of the stellar surface, so the outgoing radial flux within the disk comes very close to the Eddington limiting flux.

Overall, the transition from disk to boundary layer is much less abrupt than at lower accretion rates. For example, $\Omega$ gradually deviates from Keplerian, so that $\Omega / \Omega_{K}$ drops steadily, but $\Omega$ itself continues to increase and reaches a maximum farther in. At lower accretion rates, $\Omega$ is fairly strictly Keplerian within the disk, reaches its maximum at the boundary layer transition, and then decreases within the boundary layer. The different $\Omega$ profiles have consequences for the amount of angular momentum accreted by the star, as 
discussed below. Note that the temperature profile is quite smooth, in marked contrast to lower accretion rates. Also, the minimum in temperature at the transition disappears; this is due to heating of the inner disk by the boundary layer radiation. The height of the disk is a large fraction of the radius, $H / R \sim 0.6-0.8$, and $H / R$ stays fairly constant over much of the radial extent of the boundary layer. The boundary layer stays optically thick to scattering but optically thin to absorption.

\subsection{Viscosity Dependence}

The radial velocity of the accreting gas is approximately proportional to the viscosity coefficient, since this determines the rate at which angular momentum is removed from the gas. As a result, for a given mass accretion rate, the disk surface density is inversely proportional to viscosity. Thus, changing the value of $\alpha$ changes the optical depth of the disk.

We illustrate the effects of $\alpha$ in Fig. 10, which shows solutions with $\alpha=0.01$ and $\dot{M}=10^{-8}-10^{-10} \mathrm{M}_{\odot} \mathrm{yr}^{-1}$. In most respects this sequence looks similar to that shown in Fig. 7 above for the same accretion rates with $\alpha=0.1$. The width of the boundary layer is quite similar in the two sequences, especially at high $\dot{M}$; at $\dot{M}=10^{-10} \mathrm{M}_{\odot} \mathrm{yr}^{-1}$ the $\alpha=0.01$ boundary layer is about half as wide as it is for $\alpha=0.1$. The disk height is also quite similar - at moderate accretion rates it is slightly larger in the $\alpha=0.01$ solutions. The effective temperatures are also quite similar, as one would expect, since independent of $\alpha$, the same amount of energy is being released in an area of approximately the same size. Also as expected, the $\alpha=0.01$ solutions have lower $v_{R}$ and higher $\rho$ and $\tau_{s}$ than the $\alpha=0.1$ solutions, in all cases by a factor of $\sim 10$.

The main qualitative difference between the two sets of solutions is in the gas temperature in the boundary layer, which is always lower for the $\alpha=0.01$ solutions. The boundary layer becomes marginally optically thick for $\alpha=0.01$ at $\dot{M}=10^{-8} \mathrm{M}_{\odot} \mathrm{yr}^{-1}$, with $\tau_{*} \sim 1$; as a result, the temperature does not jump dramatically at the outer edge of the boundary layer, but increases steadily as the gas approaches the star. The $\alpha=0.01$ solutions do not show the strongly double-peaked temperature profile seen at low $\dot{M}$ for $\alpha=0.1$. Also, even at $10^{-10} \mathrm{M}_{\odot} \mathrm{yr}^{-1}$, the ion and electron temperatures are very close in the $\alpha=0.01$ solutions.

In all the solutions so far, we have used the "standard" viscosity prescription, which takes the turbulent length scale $l_{\text {turb }}=\left(H^{-2}+H_{R}^{-2}\right)^{-1 / 2}$, which is approximately equal to the lesser of the vertical and radial pressure scale heights $H$ and $H_{r}$ in the disk. If we 
compare $H$ and $H_{r}$ with the length scale for subsonic turbulence $H_{s}$, we find $H_{s}<H_{r}, H$ in much of the boundary layer, as shown in Fig. 11a for the $\dot{M}=10^{-9} \mathrm{M}_{\odot} \mathrm{yr}^{-1}, \alpha=0.1$ solution. This means that the azimuthal velocity difference across the adopted turbulent length scale is supersonic. However, note that since $\alpha=0.1$, the viscosity coefficient $\nu=\alpha c_{s} l_{\text {turb }}$ is less than the product of $c_{s}$ and the subsonic length scale $H_{s}$; in other words, over a length scale $\alpha l_{\text {turb }}$, the azimuthal velocity difference is still subsonic.

We have calculated a set of solutions using the "subsonic" viscosity prescription outlined in $\S 2$; here $l_{\text {turb }}=\left(H^{-2}+H_{R}^{-2}+H_{s}^{-2}\right)^{-1 / 2} \sim \min \left(H, H_{r}, H_{s}\right)$ (Fig. 11b). The results are shown in Fig. 12. At high $\dot{M}$, the subsonic prescription changes the solution very little, but at lower $\dot{M}$, it substantially decreases the viscosity in the boundary layer. As a result, the lower $\dot{M}$ solutions with $\alpha=0.1$ and subsonic viscosity look very much like the solutions with $\alpha=0.01$ and standard viscosity. In the boundary layer, they have lower temperatures $\left(T_{e} \sim 10^{8} \mathrm{~K}\right)$ and radial velocities and higher densities and optical depths than the standard viscosity solutions.

Solutions with subsonic viscosity can also reach higher temperatures of a few $\times 10^{8} \mathrm{~K}$ when higher values of $\alpha$ are used, as shown in Fig. 13. In the upper panels, we compare solutions with $\dot{M}=10^{-9} \mathrm{M}_{\odot} \mathrm{yr}^{-1}$ and subsonic viscosity for $\alpha=0.1$ and 0.2 to a solution with standard viscosity and $\alpha=0.1$. The solutions with subsonic viscosity reach higher boundary layer electron temperatures and lower scattering optical depths as $\alpha$ increases, and more closely resemble the solution with regular viscosity and $\alpha=0.1$. Similarly, in the lower panels we show solutions for $\dot{M}=10^{-10} \mathrm{M}_{\odot} \mathrm{yr}^{-1}$ and subsonic viscosity with $\alpha=0.1$ and 0.316 ; the electron temperature and scattering optical depth for the $\alpha=0.316$ solution are closer to the solution with standard viscosity and $\alpha=0.1$.

\subsection{Rotation and Accretion Spinup of the Star}

The coherent $\mathrm{kHz}$ oscillations recently discovered during X-ray bursts from LMXBs strongly suggest that many of these contain neutron stars which are rotating at speeds of $\sim 300 \mathrm{~Hz}$ (or possibly $\sim 600 \mathrm{~Hz}$ ) (Strohmayer et al. 1996). Rotation of the accreting star should have an important impact on the boundary layer region. The energy dissipated in the boundary layer is proportional to $\left(1-\Omega_{*} / \Omega_{K}\right)^{2}$, so it decreases rather rapidly as the star spins up.

In Fig. 14 we show the structure of the boundary layer for five different stellar rotation frequencies $f_{*}=0,318,637,1273,1910 \mathrm{~Hz}$ (corresponding to $\Omega_{*}=2 \pi f_{*}=0,2000,4000,8000,12000 \mathrm{~s}^{-1}$ ). Based on current observations, the 
lower values are the most relevant; however, the effects of rapid rotation on the boundary layer and spinup of the star are interesting enough that we have included solutions for rotation speeds extending up to nearly breakup. All of the solutions are for $\dot{M}=10^{-9} \mathrm{M}_{\odot} \mathrm{yr}^{-1}$ and $\alpha=0.1$. The width and effective temperature of the boundary layer decrease steadily as the star spins up. The low-density region becomes cooler and denser and the optical depth increases. At $\Omega_{*}=12000 \mathrm{~s}^{-1}$ the boundary layer is becoming marginally optically thick. It should be noted that rapid rotation will flatten the star and increase its equatorial radius, and we have not included this in our calculations; we do not expect that it will qualitatively change our results.

In our calculations we specify the angular momentum accretion rate $\dot{J}$. The thin disk equations assume that the angular momentum accretion rate onto the star is $\dot{J}_{\text {thin }}=\dot{M} \Omega_{K}\left(R_{*}\right) R_{*}^{2}$. The angular momentum balance then gives a factor $1-\left(R_{*} / R\right)^{1 / 2}$, which appears in the thin disk solutions and produces a maximum in the effective temperature at $R=(49 / 36) R_{*}$ (Shakura \& Sunyaev 1973). Inside this radius the effective temperature drops again, and formally goes to zero at $R=R_{*}$.

In our solutions $\dot{J} \neq \dot{J}_{\text {thin }}$. In general, $\Omega$ reaches a maximum at some radius $R_{\max }$ which is near the transition from the disk to the boundary layer, and $\dot{J}$ is set by the angular momentum of the gas at $R_{\max }, \dot{J}=\dot{M} \Omega_{\max } R_{\max }^{2}$. We specify $\dot{J}$ using the parameter $j \equiv \dot{J} / \dot{J}_{\text {thin }}$, so $j=\Omega_{\max } R_{\max }^{2} / \Omega_{K}\left(R_{*}\right) R_{*}^{2}$. If $\Omega \simeq \Omega_{K}$ in the disk and $\Omega_{\max } \simeq \Omega_{K}\left(R_{\max }\right)$, then the angular momentum equation can be solved in the usual way, except that the factor in the disk equations becomes $1-\left(R_{\max } / R\right)^{1 / 2} \simeq 1-j\left(R_{*} / R\right)^{1 / 2}$, i.e. $j \simeq\left(R_{\max } / R_{*}\right)^{1 / 2}$. The maximum in $T_{\text {eff }}$ occurs at $R \simeq(49 / 36) R_{\max } \simeq(49 / 36) j^{2} R_{*}$. In the solution shown in Figs. $3-5, j \simeq 1.064$, so $T_{\text {eff }}$ should reach a maximum at $R \simeq 1.54 R_{*}$.

The condition that the disk outside of $R_{\max }$ is close to Keplerian holds true in most of our solutions, with the exception being the solutions at high $\dot{M}$. There $\Omega$ is substantially sub-Keplerian when it reaches a maximum, and as a result the value of $j$ can be quite a bit less than $\left(R_{\max } / R_{*}\right)^{1 / 2}$. For example, our solution with $\dot{M}=10^{-8} \mathrm{M}_{\odot} \mathrm{yr}^{-1}$ shown in Fig. 7 has $j \simeq 1.155$, even though $R_{\max } \simeq 1.84 R_{*}$, because $\Omega_{\max }$ is only $\simeq 0.85$ of $\Omega_{K}\left(R_{\max }\right)$.

Note that although we specify the value of $j$, it is fairly tightly constrained. If we increase $j$, then $R_{\max }$ and the whole solution move outward in radius. We have selected values of $j$ for which the inner edge of the boundary layer, where the gas piles up on the star, is located at $R \simeq 1.035 \times 10^{6} \mathrm{~cm}$. This value is selected arbitrarily; it leaves a small zone between $R=10^{6} \mathrm{~cm}$ and $R \simeq 1.035 \times 10^{6} \mathrm{~cm}$ where the accreting gas is piling up on the star. By choosing slightly smaller or larger values of $j$, we could have located the boundary layer at a slightly smaller or larger radius. 
As an accreting star spins up near breakup, the rate of angular momentum accretion drops rapidly and becomes negative (Popham \& Narayan 1991; Paczynski 1991). We have found solutions with $j<1$, and we show a solution with $j=0$ at $\Omega_{*}=1.31 \times 10^{4} \mathrm{~s}^{-1}$ as a dashed line in Fig. 14. Here the boundary layer is absent, and the accretion flow joins the star smoothly. For larger values of $\Omega_{*}, j$ becomes negative; thus this value of $\Omega$ should be the maximum reached by the star for this choice of parameters.

\section{Discussion}

\subsection{Energetics of the Boundary Layer}

We have found that boundary layers around disk-accreting neutron stars will be hot, low in density, optically thin to absorption, and both radially and vertically extended. We begin by discussing some of the processes which are important in producing this boundary layer structure. We focus particularly on the important role played by radial energy transfer.

We find that the accreting gas makes a fairly abrupt transition from the disk to the boundary layer. The disk is thin, relatively cool and dense, and optically thick to absorption, while the boundary layer is geometrically thick, hot and rarefied, and optically thin to absorption for most choices of parameters. There is a similar abrupt transition in reverse when the hot gas nears the stellar surface. These transitions are related to the thermal instability of the hot, low-density boundary layer gas discussed by King \& Lasota (1987), in which the gas cannot efficiently radiate away the dissipated energy. However, the situation is more complicated than the one they envisioned, since they confined their analysis to local heating and cooling by dissipation and radiation, in a gas-pressure-dominated disk (corresponding to lower accretion rates and luminosities than those considered here). In our solutions radiation pressure is dominant and the energy balance is dominated not by local dissipation and radiation, but instead by Comptonization and advection.

In our solutions the gas reaches temperatures of a few $\times 10^{8} \mathrm{~K}$, and nearly reaches $10^{9}$ $\mathrm{K}$ in one case. One might expect the large energy release to make the boundary layer gas even hotter than this; if all the accretion energy were to go into heating the gas, it would reach the virial temperature of a few $\times 10^{11} \mathrm{~K}$. However, the presence of Compton cooling keeps the electron temperature from rising above $\sim 10^{9} \mathrm{~K}$. Solutions computed without Compton cooling reached very low optical depths and high temperatures in excess of $10^{9} \mathrm{~K}$ even for very small values of $\alpha=10^{-4}-10^{-3}$. For $\alpha=0.1$ these temperatures would be much higher, and would presumably approach the virial temperature. This illustrates the 
dominant role played by Comptonization in transferring energy from the gas to the photons and cooling the boundary layer region.

We have solved separate energy equations for the electrons and ions, allowing for the possibility of a two-temperature plasma. In most of our solutions, the ion and electron temperatures are essentially the same at all radii; however, in solutions with $\dot{M} \lesssim 10^{-9} \mathrm{M}_{\odot} \mathrm{yr}^{-1}$ and $\alpha \gtrsim 0.1$, the ion temperature is significantly higher than the electron temperature in the boundary layer region. The ion temperature increases rapidly as $\dot{M}$ decreases, reaching $\sim 2 \times 10^{10} \mathrm{~K}$ at $\dot{M}=10^{-10} \mathrm{M}_{\odot} \mathrm{yr}^{-1}, \alpha=0.1$. This increased ion temperature produces an increase in the gas pressure, and together with the smaller luminosity, this will lead to gas pressure becoming the dominant source of pressure at low $\dot{M}$.

Radial transport of energy by advection also plays an important role in our solutions. This is not surprising, since NP93 found that advection plays an important role in hot CV boundary layers, which led to the "rediscovery" of advection-dominated accretion by Narayan \& Yi (1994). The importance of advection can be seen simply by noting that the disk is not geometrically thin in the boundary layer region, which means that the energy density is significant compared to gravity. However, the flow in the boundary layer differs in some respects from the standard advection-dominated accretion flow (ADAF), which is heated by viscous dissipation and cooled (very inefficiently) by Compton cooling, so that essentially all the energy goes into heating the gas. In the boundary layer, as discussed in $\S 3$, the energy balance is between Compton heating (or cooling) and advection. In the outer boundary layer, the energetic photons contribute far more energy than viscous dissipation, and the gas cannot radiate this energy away, so it heats up and expands and the energy is carried inward. In the inner boundary layer, this situation is reversed. Thus, even though the situation differs somewhat from a standard ADAF, advection is nonetheless an important energy term throughout the hot region.

One of the main predictions of the mechanics of the boundary layer is that viscous transport carries energy inward, toward the dense, optically thick region at the surface of the star. The situation is very similar to that in the standard disk (Shakura \& Sunyaev 1973), where viscous transport carries energy away from the central regions of the disk and delivers it to larger radii, increasing their energy release by a factor of three compared to the simple estimate based on the the change in the gravitational and rotational energy of the infalling gas. In the boundary layer, where $d \Omega / d r$ has a different sign, the viscous torque transports energy toward the star and this leads to important consequences.

This can be seen in Figure 5, which shows the energy release due to viscous dissipation, the viscous transport of energy, and the change in kinetic energy. In the dense region in 
the innermost part of the boundary layer, the rotational velocity of the gas is much smaller than Keplerian. If we consider the deepest layer, where the radiation flux is still much smaller than the local critical Eddington flux, we see immediately that the density of the matter must be very high, because it is not supported strongly by centrifugal forces or by the radiation pressure gradient $d P_{\text {rad }} / d R \propto F_{R}$. A large fraction of the energy is dissipated in, and subsequently radiated away from, this high-density region. Much of the radiation travels radially outward into the hot, low-density region, providing the seed photons for Comptonization. In the solution shown in Figs. 3-5, the density at the point where the dissipation peaks is nearly $1 \mathrm{~g} \mathrm{~cm}^{-3}$, and the radial scattering optical depth $\tau_{s}=\int \rho \kappa_{s} d R$ between that radius and the radius where the temperature peaks is $\sim 20$, as shown in Fig. 6 . It is crucial for the formation of the spectrum that viscous transport and advection carry the bulk of the energy from the low-density, rapidly rotating outer boundary layer to the dense, slowly rotating region where the gas reaches the stellar surface.

\subsection{The Eddington Flux Limit}

The Eddington flux limit, where the outward radiation pressure gradient balances gravity, plays an important role in our solutions. For spherical accretion, $\dot{M}$ is limited to $\dot{M}_{E d d}=4 \pi c R_{*} / \kappa_{s} \simeq 1.73 \times 10^{-8} \mathrm{M}_{\odot} \mathrm{yr}^{-1}$ for $R_{*}=10^{6} \mathrm{~cm}$. In a disk geometry, where the luminosity is not emitted isotropically, the crucial quantity is the critical Eddington flux which balances the local gravitational force. The radial flux through the disk is limited to the local Eddington value $F_{E d d}=\left(G M / R^{2}\right) c / \kappa_{s}$. In the vertical direction, the downward component of gravity increases with distance $z$ from the midplane as $\sim z / R$. Thus at the disk surface $z \sim H$, the vertical flux is limited to $F_{E d d, V}=(H / R)\left(G M / R^{2}\right) c / \kappa_{s}$.

For high values of $\dot{M}$, the radial and vertical radiation fluxes approach their respective Eddington limiting fluxes. In Fig. 8 we showed that for $\dot{M}=10^{-8} \mathrm{M}_{\odot} \mathrm{yr}^{-1}$, the radial flux comes very near the Eddington value, but only over a small range of radius, and drops steadily as the radiation moves outward through the boundary layer. This reflects the fact that more of the radial support against gravity comes from the centrifugal force and less from radiation pressure as one moves outward through the boundary layer. The vertical flux, on the other hand, stays within $2 \%$ of the Eddington value throughout the entire boundary layer. Even at $\dot{M}=10^{-9} \mathrm{M}_{\odot} \mathrm{yr}^{-1}$, the vertical flux stays between $80 \%$ and $90 \%$ of $F_{E d d, V}$. This close correspondence between $F_{V}$ and $F_{E d d, V}$ means that the radiation flux from the boundary layer has a radial profile varying as $H / R^{3}$. Physically, it means that at high $\dot{M}$ the boundary layer is radiating as much flux as it can.

In order to increase $\dot{M}$, the boundary layer must radiate more energy, and in order 
to do this it must expand either radially or vertically, or both. By expanding radially, it increases the surface area through which the energy can escape, while vertical expansion increases the gravity and the limiting flux. In the outer portion of the expanded boundary layer, both the radiation flux and centrifugal forces are important in supporting matter against gravitational attraction to the neutron star. The rotational velocity decreases significantly in this region as the gas flows inward, and is replaced by radiation pressure support; however, the energy release due to viscous dissipation is very small, since as we have seen, viscous transport carries most of the energy to be dissipated farther in. Thus this region serves mostly to radiate the energy which is dissipated farther in.

We have shown that the boundary layer is both radially and vertically extended, with the radial width reaching $\sim R_{*}$ and $H / R \simeq 0.8$ at $\dot{M} \sim 10^{-8} \mathrm{M}_{\odot} \mathrm{yr}^{-1}$ (Fig. 7). If we continue to increase $\dot{M}$, the boundary layer continues to expand radially and increase its emitting area (Fig. 9). The radial expansion is quite rapid, with the boundary layer size doubling for a $40 \%$ increase in $\dot{M}$. By increasing the emitting area, the radial expansion makes it possible for the boundary layer to radiate away the very high luminosities associated with these high values of $\dot{M}$, while keeping the vertical flux below the Eddington limit.

This illustrates an important difference between disk accretion and spherical accretion. In spherical accretion, the Eddington limit is global, in the sense that any luminosity produced inside a given radius must contribute to the total outward flux at that radius. Thus the total luminosity of the system is constrained by the Eddington limit. In a disk system, the Eddington limit is local; the local radial and vertical fluxes cannot exceed the local gravity. However, there is no global limit on the luminosity, since it can be radiated away through the surface area of the boundary layer, and as we have seen, the boundary layer can expand to radiate additional luminosity as needed.

\subsection{Radiation Spectrum}

The structure of the boundary layer described above permits simple modeling of the formation of the radiation spectrum which leaves the boundary layer. We plan to calculate detailed spectra based on our solutions in a future paper; however, we can make some general statements. It is clear that Compton scattering will play a dominant role in the formation of the X-ray spectrum of the radiation which travels through the hot, low-density region. The seed photons which are Compton-scattered are primarily emitted from the denser gas which piles up at the inside edge of the hot region. The Comptonized spectrum will be characterized by $T_{e}$ and $\tau_{s}$, and especially by the Compton $y$-parameter 
$y=\left(T / T_{\text {com }}\right) \max \left(\tau_{s}, \tau_{s}^{2}\right)$.

These parameters vary with radius in our solutions, and of course $T_{e}$ and $\rho$ also vary with vertical position in the disk, and this variation is not included directly in our solutions. Also, $\tau_{s}=\kappa_{s} \rho H$ in the vertical optical depth for our solutions, but the photons are not travelling only vertically, but also radially and azimuthally, and being scattered in all directions, so the path traveled by a given photon may be much longer than $H$. Therefore, although we can get a rough sense of what the X-ray spectrum will look like based on the characteristic values of $T_{e}$ and $\tau_{s}$ for a given solution, the formation of the real spectrum will be considerably more complex.

In the optically thick region, where Thompson scattering nevertheless strongly dominates the opacity, free-free processes easily produce many photons at low frequencies where they $\left(K_{\nu} \sim \nu^{-2}\right)$ are more effective than Comptonization. Comptonization increases the energy of low frequency photons due to the Doppler effect and leads to the diffusion of the photons towards higher frequencies. At some frequency $x_{0}=\frac{h \nu_{0}}{k T_{e}}$ the rate at which Comptonization can take photons and bring them to higher frequencies equals the rate of photon absorption due to bremsstrahlung. Practically every photon born with a frequency higher than $x_{0}$ will be transported toward the frequency $x=3$ due to the Comptonization process. This picture is very similar to the processes occurring in the early universe and is described in detail by Illarionov \& Sunyaev (1975). Comptonization is very effective because the parameter $y=\frac{k T_{e}}{m_{e} c^{2}} \tau_{s}^{2} \gg 1$. Under these circumstances a Wien-type spectrum must be formed when $x_{0}$ is very small and $y \gtrsim 1$ but not very high. At higher $\tau_{s}$ and $y$ a Bose-Einstein spectrum is formed and the formation of the black body spectrum is possible inside very deep regions.

Radiation with this spectrum diffuses out from the dense regions toward more and more rarified regions, where the production of new soft photons becomes more and more difficult due to the low density. Comptonization continues to dominate, and therefore the spectrum tends to be close to a Wien spectrum, with a strong increase of intensity at low frequencies where the spectrum becomes a Rayleigh-Jeans spectrum. The temperature of electrons in the outer regions is determined by the radiation: high energy photons heat electrons due to the recoil effect, and low energy photons cool them down. (See Levich \& Sunyaev 1971). This process is illustrated by Fig. 5. We see that Comptonization takes energy from the plasma in the inner part of the boundary layer and gives energy back to the plasma in the outer part, heating the electrons. As a result, we are producing a quasi-Wien-type radiation spectrum with strong low frequency excess, and radiation with such a spectrum escapes from every point of the surface of the boundary layer. Different regions of the boundary layer have a range of temperatures, depending on the distance 
from both the stellar surface and from the midplane of the disk. Therefore we will observe a spectrum which is a sum of Wien spectra with different temperatures.

The picture described above follows the results of extended calculations by Grebenev \& Sunyaev (2000) for the spreading layer model (Inogamov \& Sunyaev 1999) of the surface of the neutron star. It is interesting to note that this "spreading layer" picture of the boundary layer, in which the gas loses angular momentum as it spreads over the stellar surface, gives rather similar results to the ones presented here. In both cases, the size of the layer increases as the mass accretion rate and luminosity increase. The "spreading layer" has a meridional extent of about $0.45 \mathrm{~km}$ at $L / L_{E}=0.01$ (which should correspond approximately to our $\dot{M}=10^{-10} \mathrm{M}_{\odot} \mathrm{yr}^{-1}$ solutions), increasing to $\sim 17 \mathrm{~km}$ at $L / L_{E}=0.8$. In our solutions the radial extent of the boundary layer is $\sim 1,0.5 \mathrm{~km}$ for $\dot{M}=10^{-10} \mathrm{M}_{\odot} \mathrm{yr}^{-1}$ and $\alpha=0.1,0.01$, respectively, and $\sim 10 \mathrm{~km}$ for $\dot{M}=10^{-8} \mathrm{M}_{\odot} \mathrm{yr}^{-1}$. In addition, the optical depths and $y$-parameters are quite similar in the two types of solutions. Inogamov \& Sunyaev (1999) found $\tau_{s} \sim 3$ at $L / L_{E}=0.01$ and $\tau_{s} \sim 1000, y \sim 10^{5}$ at $L / L_{E}=0.8$, quite similar to the values presented above. Thus the two treatments should result in rather similar spectra. Most importantly, in both cases the majority of the energy release occurs in a dense region which is covered by a levitating low density region where the final spectrum is formed. The agreement is remarkable considering that the accretion flow is treated quite differently in the two approaches.

The question of which approach is the correct one is difficult to answer at present. The main difference between them is the assumed geometry of the boundary layer region. The "spreading layer" treatment assumes that the disk material enters the spreading layer at nearly Keplerian rotation velocity and small disk height; i.e., very little angular momentum is lost in the disk and there is no disk boundary layer of the type calculated in the present paper. Conversely, the approach taken here assumes that the drop from Keplerian to the stellar angular velocity takes place during the radial inflow of the gas, rather than during the spreading of the accreted gas over the stellar surface. A multi-dimensional treatment will be required to distinguish between these two possibilities. The treatment of viscosity in the disk and surface layer may have an important impact on the results. Note that the viscosity prescription adopted by Inogamov \& Sunyaev (1999) is also quite different from the one used here; the viscosity decreases as the gas approaches the neutron star surface in analogy with the behavior of fluid near a wall in laboratory experiments. Yet despite these differences their results are rather similar to ours. 


\subsection{Comparison to Observed Spectra of LMXBs}

Most observed LMXBs have been in one of two spectral states: a low state characterized by low luminosity and a hard, power-law spectrum, or a high state characterized by high luminosity and a softer spectrum. For example, the four LMXBs recently observed by Barret et al. (1999) included three in the low state and one in the high state. The three low state sources 1E1724-3045, GS1826-238, and SLX1735-269, all had 1 - 200keV luminosities of $\sim 1-1.5 \times 10^{37} \mathrm{erg} \mathrm{s}^{-1}$, and their spectra were fitted by thermal Comptonization by gas with electron temperatures of $\sim 25-30 \mathrm{keVand}$ optical depths of a few. The high state source KS1731-260 had $L(1-200 \mathrm{keV}) \simeq 8-9 \times 10^{37} \mathrm{erg} \mathrm{s}^{-1}$, and was fitted by a much softer Comptonized spectrum with $T_{e} \sim 2.6-2.8 \mathrm{keV}$ and $\tau \sim 10$.

Our $\dot{M}=10^{-10}$ solution with $\alpha=0.1$ and regular viscosity (shown in Fig. 7 ) has $T_{e}$ in the hot boundary layer varying from $\sim 10^{8}-10^{9} \mathrm{~K}$ and $\tau_{s} \sim 0.5-1$, giving a Compton $y$-parameter of less than 1 . The large variations in $T_{e}$ and $\tau_{s}$ make it difficult to predict what the spectrum will look like, but probably it will have a general power-law shape, and may extend to rather high energies due to the very high $T_{e}$ in the hottest part of the boundary layer.

At moderate $\dot{M} \sim 10^{-9} \mathrm{M}_{\odot} \mathrm{yr}^{-1}, T_{e} \sim 2-3 \times 10^{8} \mathrm{~K}$ and both $\tau_{s}$ and $y \sim$ a few. Unsaturated Comptonization in the hot boundary layer should produce a power-law spectrum with a cutoff at $\sim 20-30 \mathrm{keV}$. This solution has a total luminosity of $\sim 10^{37} \mathrm{erg} \mathrm{s}^{-1}$, which corresponds approximately to that of the low-state LMXBs oberved by Barret et al. (1999), and the values of $T_{e}$ and $\tau_{s}$ agree well with those from their fits.

At high $\dot{M}, \tau_{s}$ is in the hundreds (for $\alpha=0.1$ ) or thousands (for $\alpha=0.01$ ) and $y \sim 10^{5}$. The optical depth for combined absorption and scattering is $\tau_{*} \sim 0.1$ for $\alpha=0.01$ but only $\tau_{*} \sim 0.001$ for $\alpha=0.1$. Saturated Comptonization will then produce a Wien spectrum which peaks at $\sim 3 k T$ (Illarionov \& Sunyaev 1975). $T$ varies from $\sim 10^{8} \mathrm{~K}$ for $\alpha=0.01$ to $\sim 3 \times 10^{8} \mathrm{~K}$ for $\alpha=0.1$. This will produce spectra with $k T \sim 10-30 \mathrm{keV}$. However, these are the spectra that will be produced by the gas near the disk midplane, but these solutions have such large values of $\tau_{s}$ and $y$ that we expect a substantial temperature gradient, with the gas near the surface much cooler than at the midplane. Thus the spectrum will strongly resemble a blackbody spectrum with a characteristic temperature which is close to the effective temperature of the gas rather than the midplane temperature, i.e. around 1.5-2 keV. Blackbody fits to luminous LMXBs (e.g. Mitsuda et al. 1984) give $k T \sim 2 \mathrm{keV}$, while White et al. (1986) used a $1-2 \mathrm{keV}$ blackbody plus an unsaturated Comptonized component with a cutoff at $3-8 \mathrm{keV}$.

Overall, it appears that our solutions should fit the spectral data reasonably well. The 
variation of boundary layer temperature with $\dot{M}$ depends on $\alpha$; for low $\dot{M}$, the boundary layer is much hotter for $\alpha=0.1$ than for $\alpha=0.01$. Since low-luminosity LMXBs generally are observed to have rather hard power-law tails which are fit using electron temperatures of $\sim 30 \mathrm{keV}$, it seems that our models will agree with the observations better if a large value of $\alpha$ is used.

X-ray spectra of LMXBs frequently show evidence for iron line emission at about 6.4 $\mathrm{keV}$, which is believed to result from X-ray irradiation of the surface of the disk. In our solutions, the hot boundary layer is much thicker than the inner disk, so the disk surface should intercept a reasonable fraction of the X-ray emission. This fraction will depend on the boundary layer thickness and radial extent and the disk height profile. Fig. 1 shows the height profile of the boundary layer and disk for four solutions. The solution with $\dot{M}=10^{-8} \mathrm{M}_{\odot} \mathrm{yr}^{-1}$ has a thicker boundary layer and disk than the $\dot{M}=10^{-9} \mathrm{M}_{\odot} \mathrm{yr}^{-1}$ or $10^{-10} \mathrm{M}_{\odot} \mathrm{yr}^{-1}$ solutions, so a larger fraction of the boundary layer emission should be intercepted by the disk. The solution for a rotating neutron star has a thinner boundary layer than for the nonrotating star. Note that the axes are chosen to emphasize the differences between the three solutions and give a distorted impression of the shape of the disk; in fact the disk is quite thin, with $H / R \sim 0.01-0.02$ at all radii. Thus the disk is basically flat, and the fraction of the boundary layer emission intercepted by the disk will be around 25\% (Lapidus \& Sunyaev 1985). The stellar surface will also intercept a substantial fraction of the X-ray emission (Popham 1997), but most of this will be reradiated back into the hot boundary layer gas. The X-ray flux incident on the disk and star will result in a number of interesting effects, including polarization (Lapidus \& Sunyaev 1985), line emission, and a "Compton reflection" spectrum.

\subsection{Implications for Multicomponent Spectral Fits}

With a self-consistent picture of the dynamics and energetics of the boundary layer region, we are now in a position to assess the multi-component models commonly used to fit LMXB spectra. In particular, we can discuss the emitting regions which are present. The first component, present in most of our solutions, is a hot, low-density boundary layer region which cools by inverse-Compton scattering of photons emitted from the cooler, optically thick zone near the stellar surface. The hot region also emits bremsstrahlung radiation, but in our solutions this is an insignificantly small fraction of the total emission. The temperature and density of the gas vary across the boundary layer, so the use of a single-temperature Comptonizing cloud will only approximate the true emitted spectrum. This temperature variation is quite pronounced at low $\dot{M}$ for $\alpha=0.1$; for other choices of 
parameters the single-temperature approximation may not be so bad.

The second component is the disk, which is optically thick due to the combined effects of absorption and scattering. The scattering opacity is much larger than the absorptive opacity in our solutions, so the disk should emit a modified blackbody spectrum. In general the effective temperature of the inner disk is in the range $3-6 \times 10^{6} \mathrm{~K}$, so $k T \simeq 0.25-0.5$ $\mathrm{keV}$, but the color temperature of the disk radiation will be higher due to the modified blackbody spectrum. In multi-component models, the "multi-color disk" component is often parameterized using an inner radius $r_{i n}$; in this context it is useful to note that the inner disk radius in our solutions is set by the radial extent of the boundary layer, and it varies by a factor of two as $\dot{M}$ changes.

Two-component models which consist of a Comptonized blackbody spectrum plus a modified blackbody multi-color disk would provide the best approximation to the solutions shown here. The two components would be constrained to having the appropriate luminosities. For a narrow boundary layer around a non-rotating star, the disk and boundary layer each contribute half of the total accretion luminosity $L_{a c c} \equiv G M \dot{M} / R_{*}$. The disk luminosity can be substantially lower than this, since the inner radius of the disk can be as much as $\sim 2 R_{*}$. The boundary layer luminosity is much less than $L_{a c c} / 2$ if the accreting star is rotating in the same direction as the disk, which it should be due to accretion spinup. The total luminosity of the boundary layer and disk varies as $1-j f+0.5 f^{2}$, where

$f \equiv \Omega_{*} / \Omega_{K}\left(R_{*}\right)$ is the spin rate of the star as a fraction of the breakup rate (PN95). If the outer edge of the boundary layer is at a radius $b R_{*}$, then $j \simeq b^{1 / 2}$. The disk and boundary layer luminosities will then be

$$
L_{d i s k} \simeq L_{a c c}\left(\frac{1}{2 b}\right) \quad L_{b l} \simeq L_{a c c}\left(1-b^{1 / 2} f+\frac{f^{2}}{2}-\frac{1}{2 b}\right) .
$$

By using two components with the correct temperatures and optical depths and these luminosities, it should be possible to produce a reasonable approximation to the spectrum that would be emitted by our solutions.

\subsection{Comparison with Boundary Layers in CVs and Implications for Oscillations}

In many respects the boundary layer solutions for accreting neutron stars in this paper resemble the solutions for very hot boundary layers in CVs presented by NP93. In both CVs and LMXBs, the radial extent of the boundary layer can be comparable to the stellar radius. One difference between the two types of solutions is that the radial extent of the 
boundary layer generally increases with increasing $\dot{M}$ for LMXB solutions, while it increases with decreasing $\dot{M}$ for CV solutions. Both types of boundary layers reach high temperatures $T \sim 10^{8}$, but in some cases the LMXB solutions get quite a bit hotter than this. One might expect the LMXB boundary layers to be much hotter than the CV ones, but as discussed above, Compton cooling limits the temperature. The CV boundary layer solutions of NP93 have $\tau_{s}<1$ and $T_{e} \sim 10^{8} \mathrm{~K}$, so the effects of Compton cooling should be small.

One important observational characteristic of LMXBs is the presence of $\mathrm{kHz}$ QPOs. One of us has argued that $\mathrm{kHz}$ QPOs in LMXBs are very similar to dwarf nova oscillations (DNOs) observed in CVs, and that DNOs could arise at the boundary between the disk and the hot, low-density boundary layer (Popham 1999, 2000). Since we have shown here that LMXBs should have hot, low-density boundary layers similar to those in CVs, the logical next step is that $\mathrm{kHz}$ QPOs could arise at this same location. In our solutions the disk-boundary layer transition occurs at $\sim 1.1-2.0 R_{*}$, moving outward as $\dot{M}$ increases from $10^{-10}$ to $10^{-8} \mathrm{M}_{\odot} \mathrm{yr}^{-1}$. The Keplerian rotation frequencies for this range of radii are $\sim 770-1880 \mathrm{~Hz}$ for our choice of the neutron star mass $\left(1.4 \mathrm{M}_{\odot}\right)$ and radius $(10 \mathrm{~km})$. For a larger neutron star radius, e.g. $13 \mathrm{~km}$, the range would be $\sim 520-1270 \mathrm{~Hz}$, which matches the observed range of frequencies quite well.

One difficulty with this picture is that unlike the CV solutions (NP93), our LMXB solutions have this transition radius increasing with increasing $\dot{M}$. If the oscillation period is just the Keplerian period at the transition radius, it should also increase with increasing $\dot{M}$. However, in the oscillations observed thus far, as the oscillation period increases, $\dot{M}$ is inferred to decrease. Thus, if $\mathrm{kHz}$ QPOs are formed at the disk-boundary layer transition, either $\dot{M}$ must change in the opposite sense to that inferred from the observations, or the change in the transition radius with $\dot{M}$ must be opposite to what our models predict. Note that the transition radius reaches a minimum for $\dot{M}<10^{-9.8} \mathrm{M}_{\odot} \mathrm{yr}^{-1}$ (for $\alpha=0.1$ ), and then begins to move back out again as $\dot{M}$ decreases. Thus for low values of $\dot{M}$, the sense of the variation of the Keplerian period with $\dot{M}$ would agree with the $\mathrm{kHz}$ QPO observations; however, these values of $\dot{M}$ correspond to lower luminosities than are observed from the systems which show $\mathrm{kHz}$ QPOs. For higher values of $\alpha$, the transition radius might turn around at a higher $\dot{M}$, and when we add additional physical effects to our model, the dependence of the transition radius on $\dot{M}$ may change.

\subsection{Limitations of the Model and Future Improvements}

One obvious limitation of the model used here is the use of one-dimensional, vertically-averaged equations to model the boundary layer region. This region is inherently 
two-dimensional, and by using one-dimensional equations, we are unable to simulate a number of aspects of the flow of matter and radiation. Most of the important physical quantities are assumed to be constant with height $z$ above the midplane, but in reality they vary with both $R$ and $z$. The viscous dissipation rate varies with $z$, and the processes which produce radial energy transport will also produce vertical energy transport. As discussed in $\S 2$, the abrupt drop in the disk height $H$ just before the gas reaches the stellar surface may also be a consequence of our use of simplified one-dimensional equations. Finally, the main differences between the model presented here and the "spreading layer" model of the flow of the gas over the neutron star surface are different assumptions about the geometry of the gas as it reaches the star. By constructing a two-dimensional model, we could eliminate a number of these problems.

Another important improvement to be made to the model is the inclusion of general relativistic effects. We have found infall velocities $v_{R}$ which are at most $\sim 0.01 c$, but the accreting gas may reach much larger infall velocities if it falls inside the marginally stable orbit. In addition to purely dynamical effects, radiation drag can remove angular momentum from the gas (Miller \& Lamb 1993, 1996).

Our current equations for radiative transfer are also rather crude. In particular, the assumption of frequency independence makes our treatment of absorption and of Compton scattering very approximate. Also, in our current form of the radiative transfer equations, all radial flux stays inside the disk, except that which is scattered or absorbed and reemitted as vertical flux. This is reasonable when the disk height varies slowly with radius, but in our boundary layers the disk height varies rapidly (see Fig. 1). This should be taken into account; for instance, some of the outward radial flux should escape from the outer side of the boundary layer as "vertical" flux when the disk height drops rapidly there. Since radiation pressure is important in supporting the gas, this could affect the size of the boundary layer region.

\section{REFERENCES}

Abramowicz, M. A., Czerny, B., Lasota, J.-P., \& Szuszkiewicz, E. 1988, ApJ, 332, 646

Abramowicz, M. A., Igumenshchev, I. V., \& Lasota, J.-P. 1998, MNRAS, 293, 443

Balbus, S. A., \& Hawley, J. F. 1997, in Accretion Phenomena and Related Outflows, ed. D. Wickramasinghe et al. (San Franciso: Astronomical Society of the Pacific), p. 90

Barret, D., Olive, D. F., Boirin, L., Done, C., Skinner, G. K., \& Grindlay, J. G. 1999, ApJ, in press, astro-ph/9911042 
Barret, D., \& Vedrenne, G. 1994, ApJS, 92, 505

Basko, M. M., \& Sunyaev, R. A. 1976, MNRAS, 175, 395

Chandrasekhar, S. 1960, Proc. N. A. S., 46, 53

Ghosh, P., Lamb, F. K., \& Pethick, C. J. 1977, ApJ, 217, 578

Giacconi, R., et al. 1962, Phys. Rev. Lett., 9, 439

Grebenev, S., \& Sunyaev, R. A., 2000, in preparation

Hasinger, G., \& van der Klis, M. 1989, A\&A, 225, 79

Illarionov, A. F., \& Sunyaev, R. A. 1975, Sov. Astron., 18, 413

Inogamov, N. A., \& Sunyaev, R. A. 1999, Astron. Lett., 25, 269 (astro-ph/9904333)

Joseph, D. D. 1976, Stability of Fluid Motions (Berlin: Springer)

King, A. R., Lasota, J. P. 1987, A\&A, 185, 155

King, A. R., Shaviv, G. 1984, Nature, 308, 519

Kley, W. 1991, A\&A, 247, 95

Kluźniak, W., \& Wilson, J. R. 1991, ApJ, 372, L87

Landau, L. D., \& Lifshitz, E. M. 1959, Fluid Mechanics (Oxford: Pergamon)

Lapidus, I. I., \& Sunyaev, R. A. 1985, MNRAS, 217, 291

Middleditch, J., \& Priedhorsky, W. C. 1986, ApJ, 306, 230

Miller, M. C., \& Lamb, F. K. 1993, ApJ, 413, L43

Miller, M. C., \& Lamb, F. K. 1996, ApJ, 470, 1033

Mitsuda, K., et al. 1984, PASJ, 36, 741

Muchotrzeb, B., \& Paczyński, B. 1982, Acta Astr., 32, 1

Narayan, R. 1992, ApJ, 394, 261

Narayan, R., Loeb, A., \& Kumar, S. 1994, ApJ, 431, 359

Narayan, R., \& Popham, R. 1993, Nature, 362, 820 (NP93) 
Paczyński, B. 1991, ApJ, 370, 597

Paczyński, B., \& Bisnovatyi-Kogan, G. 1981, 31, 283

Papaloizou, J. C. B., \& Stanley, G. Q. G. 1986, MNRAS, 220, 593

Patterson, J., \& Raymond, J. C. 1985, ApJ, 292, 535

Popham, R. 1997, ApJ, 478, 734

Popham, R. 1999a, MNRAS, 308, 979

Popham, R. 1999b, in preparation

Popham, R., Kenyon, S., Hartmann, L., \& Narayan, R. 1996, ApJ, 473, 422

Popham, R., \& Narayan, R. 1991, ApJ, 370, 604

Popham, R., \& Narayan, R. 1995, ApJ, 442, 337 (PN95)

Popham, R., Narayan, R., Hartmann, L., \& Kenyon, S. 1993, ApJ, 415, L127

Pringle, J. E. 1977, MNRAS, 178, 195

Pringle, J. E., \& Rees, M. J. 1972, A\&A, 21, 1

Pringle, J. E., \& Savonije, G. J. 1979, MNRAS, 187, 777

Rayleigh, Lord. 1916, Proc. Roy. Soc. A, 93, 148

Schlichting, H. 1960, Boundary Layer Theory (New York: McGraw-Hill)

Shakura, N. I., \& Sunyaev, R. A. 1973, A\&A, 24, 337

Shakura, N. I., \& Sunyaev, R. A. 1988, Adv. Sp. Res., 8(2), 135

Sibgatullin, N. R., \& Sunyaev, R. A. 1998, Astron. Lett., 24, 774 astro-ph/9811028)

Spitzer, L. 1962, Physics of Fully Ionized Gases (2nd ed.; New York: Wiley)

Strohmayer, T. E., Zhang, W., Swank, J. H., Smale, A., Titarchuk, L., Day, C., \& Lee, U. 1996, ApJ, 469, L9

Sunyaev, R. A., \& Shakura, N. I. 1986, Sov. Astron. Lett., 12, 117

Sunyaev, R. A., \& Titarchuk, L. G. 1980, A\&A, 86, 121 
Trümper, J., Pietsch, W., Reppin, C., Voges, W., Staubert, R., \& Kendziorra, E. 1978, ApJ, 219, L105

Tylenda, R. 1981, Acta Astron., 31, 267

van der Klis, M. 1998, preprint, to appear in the Proceedings of the Third William Fairbank Meeting, astro-ph/9812395

van der Klis, M., Jansen, F., van Paradijs, J., Lewin, W. H. G., van den Heuvel, E. P. J., Trümper, J., \& Szatjno, M. 1985, Nature, 316, 225

Velikhov, E. P. 1959, Sov. Phys. - JETP, 36, 995

Weinberg, S. 1972, Gravitation and Cosmology (New York: Wiley)

White, N. E., Peacock, A., Hasinger, G., Mason, K. O., Manzo, G., Taylor, B. G., \& Branduardi-Raymont, G. 1986, MNRAS, 218, 129

White, N. E., Stella, L., \& Parmar, A. N. 1988, ApJ, 324, 363 
Fig. 1.- The vertical pressure scale height $H$ (top) and the angular velocity $\Omega$ (bottom), for solutions with $\dot{M}=10^{-10}$ (long-dashed), $10^{-9}$ (solid) and $10^{-8} \mathrm{M}_{\odot} \mathrm{yr}^{-1}$ (dotted), all for a non-rotating neutron star, and for $\dot{M}=10^{-9} \mathrm{M}_{\odot} \mathrm{yr}^{-1}$ and a neutron star rotation frequency $f_{*}=636 \mathrm{~Hz}$ (dashed), all with standard viscosity and $\alpha=0.1$. Note the very small values of $H$ at the "neck" between the disk and boundary layer in the lower $\dot{M}$ solutions, and rapid increase in $H$ in the boundary layer.

Fig. 2.- The Reynolds number $R e=\Omega R\left(R-R_{*}\right) / \nu_{\text {rad }}$ calculated from our solutions, but assuming that the viscosity is given by the radiative viscosity $\nu_{\text {rad }}$.

Fig. 3.- Angular velocity $\Omega$ (solid) and Keplerian angular velocity $\Omega_{K}$ (dashed), radial velocity $v_{r}$ (solid) and sound speed $c_{s}$ (dashed), electron temperature $T_{e}$, effective temperature $T_{\text {eff }}$, density $\rho$, vertical pressure scale height $H$, vertical scattering optical depth $\tau_{s}$, and vertical effective optical depth $\tau_{*}$, for a solution with $\dot{M}=10^{-9} \mathrm{M}_{\odot} \mathrm{yr}^{-1}$, $\alpha=0.1, \Omega_{*}=0$, and standard viscosity.

Fig. 4.- Additional quantities for the solution shown in Fig. 3. (a) Total pressure (solid) and radiation pressure (dashed)in cgs units. (b) The fraction $\beta$ of the total pressure which is due to gas pressure. (c) Mean intensity of radiation $u$ (solid) and blackbody intensity $B=(\sigma / \pi) T_{e}^{4}$ (dashed) in cgs units. (d) Electron temperature $T_{e}$ (solid), photon temperature $T_{\text {phot }}$ (dashed), and ion temperature $T_{i}$ (dotted). (e) The radial flux of radiation at the disk midplane (solid) and the vertical flux at the disk surface (dotted), in units of $10^{25} \mathrm{erg} \mathrm{cm}^{-2} \mathrm{~s}^{-1}$. (f) The ratio of the radial to the vertical flux, expressed as the angle $\tan ^{-1}\left(F_{R} / F_{V}\right)$ in degrees (solid), so that zero corresponds to vertical and 90 degrees to radial. The small arrows show the angle graphically, and the dotted line is the disk height $H$ (units shown on right axis).

Fig. 5.- (Top row) Radial acceleration due to gravity (solid), centrifugal acceleration (dotted), pressure gradient (dashed), bulk viscosity (long-dashed), and radial velocity gradient (dot-dashed) in units of $10^{14} \mathrm{~cm} \mathrm{~s}^{-2}$. (Second row) Energy gain or loss per unit disk midplane area in the outer boundary layer: advection (solid), Comptonization (longdashed), viscous dissipation (dotted), radiation (short-dashed), in units of $10^{25} \mathrm{erg} \mathrm{cm}^{-2} \mathrm{~s}^{-1}$. (Third row) Viscous transport of energy in the boundary layer and disk (upper panels), where positive values correspond to outward radial transport. (Bottom row) The energy deposited or removed from the disk, normalized by the local gravitational energy release (lower panels), by viscous dissipation (solid), change in rotational kinetic energy (dotted), and viscous transport (dashed).

Fig. 6. - The energy deposited or removed from the disk, normalized by the local gravitational energy release, as a function of (top) the radial optical depth measured along 
the midplane, and (bottom) the vertical optical depth from the midplane to the surface. For the radial optical depth, $\tau=0$ corresponds to the point where $d \Omega / d R=0$, and $\tau$ is increasing inward. The upper axes shows some corresponding values of the radius $R$. Due to the viscous transport of energy, energy is dissipated where $\tau$ is large.

Fig. 7. - The change in the boundary layer structure as a function of the mass accretion rate $\dot{M}$ for five solutions with $\dot{M}=10^{-8}, 10^{-8.5}, 10^{-9}, 10^{-9.5}$, and $10^{-10} \mathrm{M}_{\odot} \mathrm{yr}^{-1}$.

Fig. 8. - The Eddington radial flux (dotted) and the radial flux for the $\dot{M}=10^{-8} \mathrm{M}_{\odot} \mathrm{yr}^{-1}$ (solid), $10^{-9} \mathrm{M}_{\odot} \mathrm{yr}^{-1}$ (short-dashed), and $10^{-10} \mathrm{M}_{\odot} \mathrm{yr}^{-1}$ (long-dashed) solutions.

Fig. 9.- The change in the boundary layer structure with $\dot{M}$, for values of $\dot{M}$ near the Eddington limit: $\operatorname{dot} M=10^{-8}, 10^{-7.95}, 10^{-7.90}$, and $10^{-7.85} \mathrm{M}_{\odot} \mathrm{yr}^{-1}$.

Fig. 10. - Same as Fig. 7, but for $\alpha=0.01$.

Fig. 11. - (a) The turbulent length scale $l_{\text {turb }}=\left(H^{-2}+H_{r}^{-2}\right)^{-1 / 2}$ (solid), the vertical pressure scale height $H$ (dotted), the radial pressure scale height $H_{r}$ (short-dashed), and the subsonic length scale $H_{s}$ (long-dashed) for the solution with $\dot{M}=10^{-9} \mathrm{M}_{\odot} \mathrm{yr}^{-1}, \alpha=0.1, \Omega_{*}=0$, and standard viscosity. In the boundary layer, $l_{\text {turb }}>H_{s}$. (b) Same as (a), but for a solution with subsonic viscosity.

Fig. 12.- Same as Fig. 7, but for "subsonic" viscosity (see text) with $\alpha=0.1$.

Fig. 13.- Comparison of the electron temperature $T_{e}$ and the vertical scattering optical depth $\tau_{s}$ for solutions with regular and subsonic viscosity. Solutions in the top panels have $\dot{M}=10^{-9} \mathrm{M}_{\odot} \mathrm{yr}^{-1}$ and subsonic viscosity with $\alpha=0.1$ (dotted), 0.2 (dashed), or standard viscosity with $\alpha=0.1$ (solid); those in the bottom panels have $\dot{M}=10^{-10} \mathrm{M}_{\odot} \mathrm{yr}^{-1}$ and subsonic viscosity with $\alpha=0.1$ (dotted), 0.316 (dashed), or regular viscosity with $\alpha=0.1$ (solid).

Fig. 14. - The change in the boundary layer structure with the rotation rate of the accreting neutron star for five solutions with $f_{*}=318,637,1273,1910 \mathrm{~Hz}\left(\Omega_{*}=0,2000,4000,8000\right.$, and $12000 \mathrm{~s}^{-1} \cdot \dot{M}=10^{-9} \mathrm{M}_{\odot} \mathrm{yr}^{-1}$ and $\alpha=0.1$ for all solutions. The dashed line is a solution with $f_{*}=2085 \mathrm{~Hz}\left(\Omega_{*}=13100 \mathrm{~s}^{-1}\right)$ and $j=0$. 


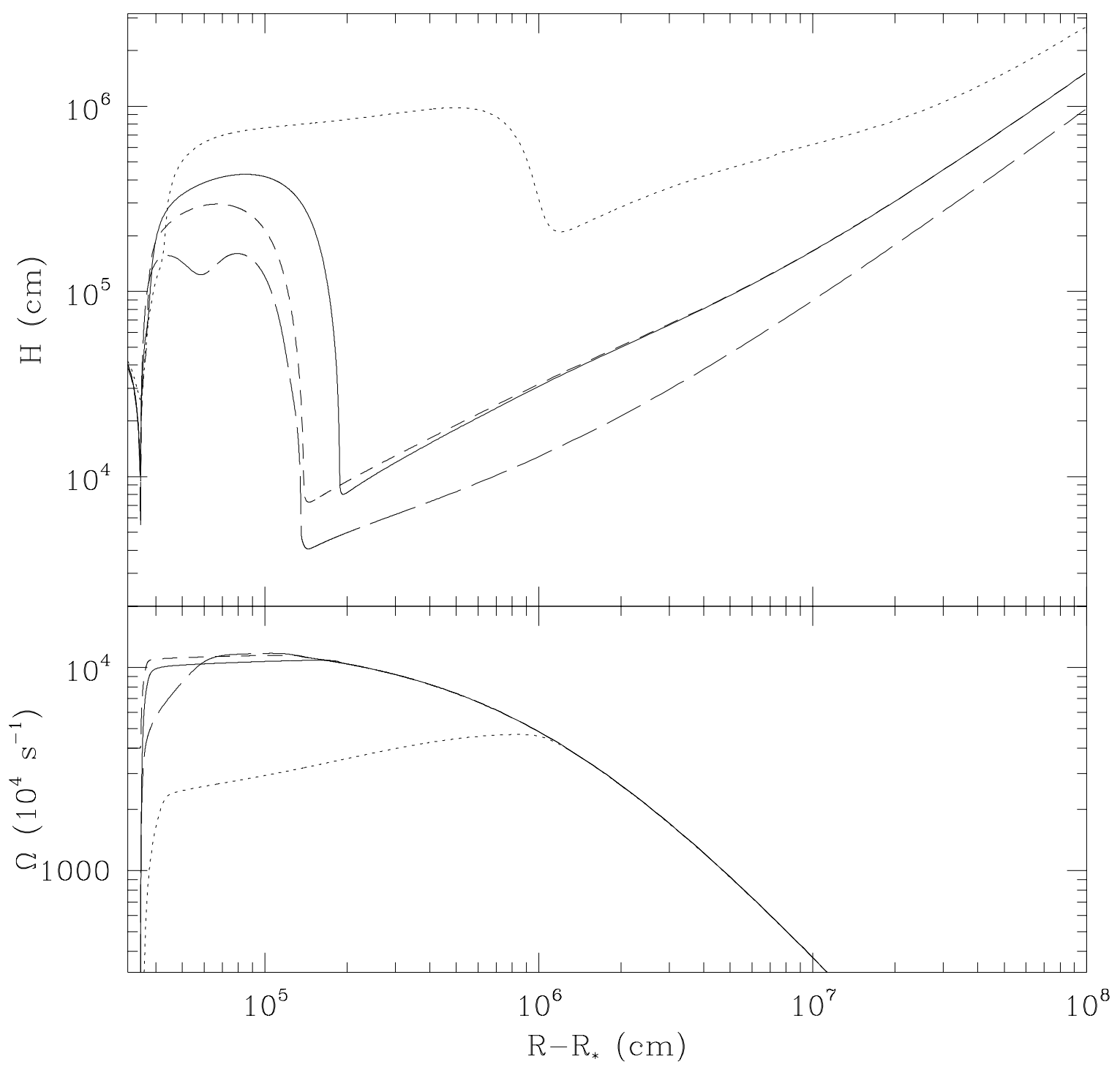




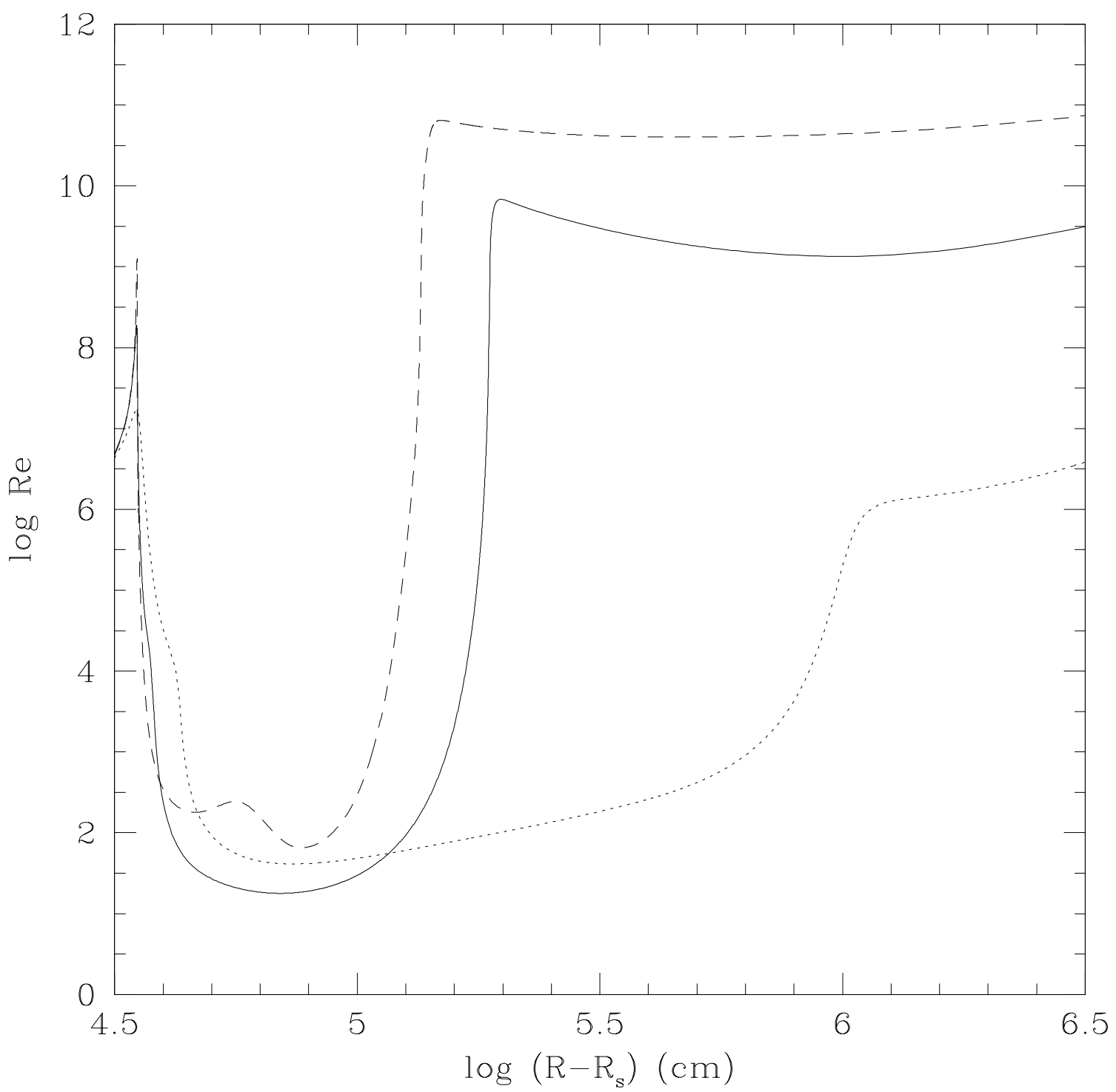



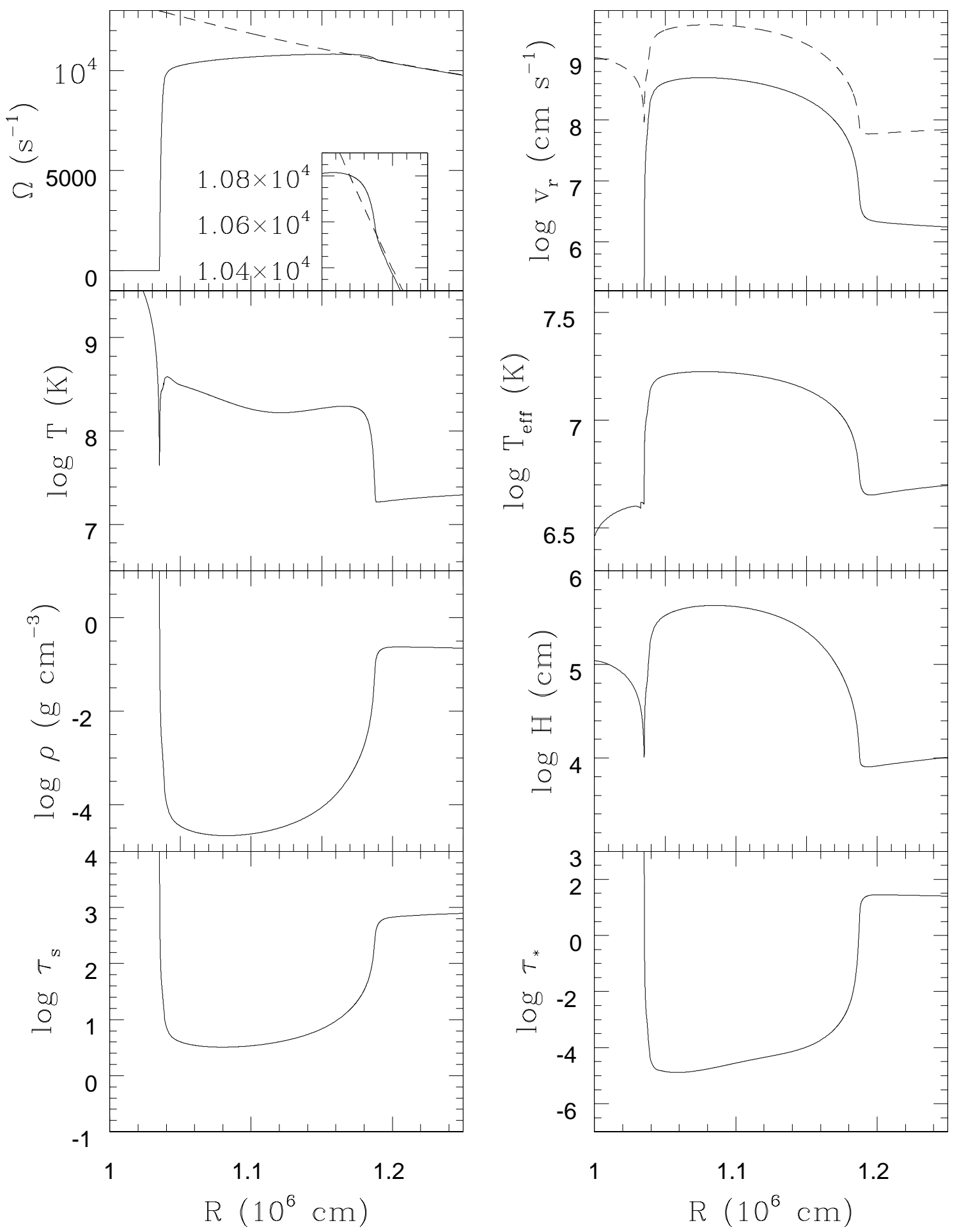

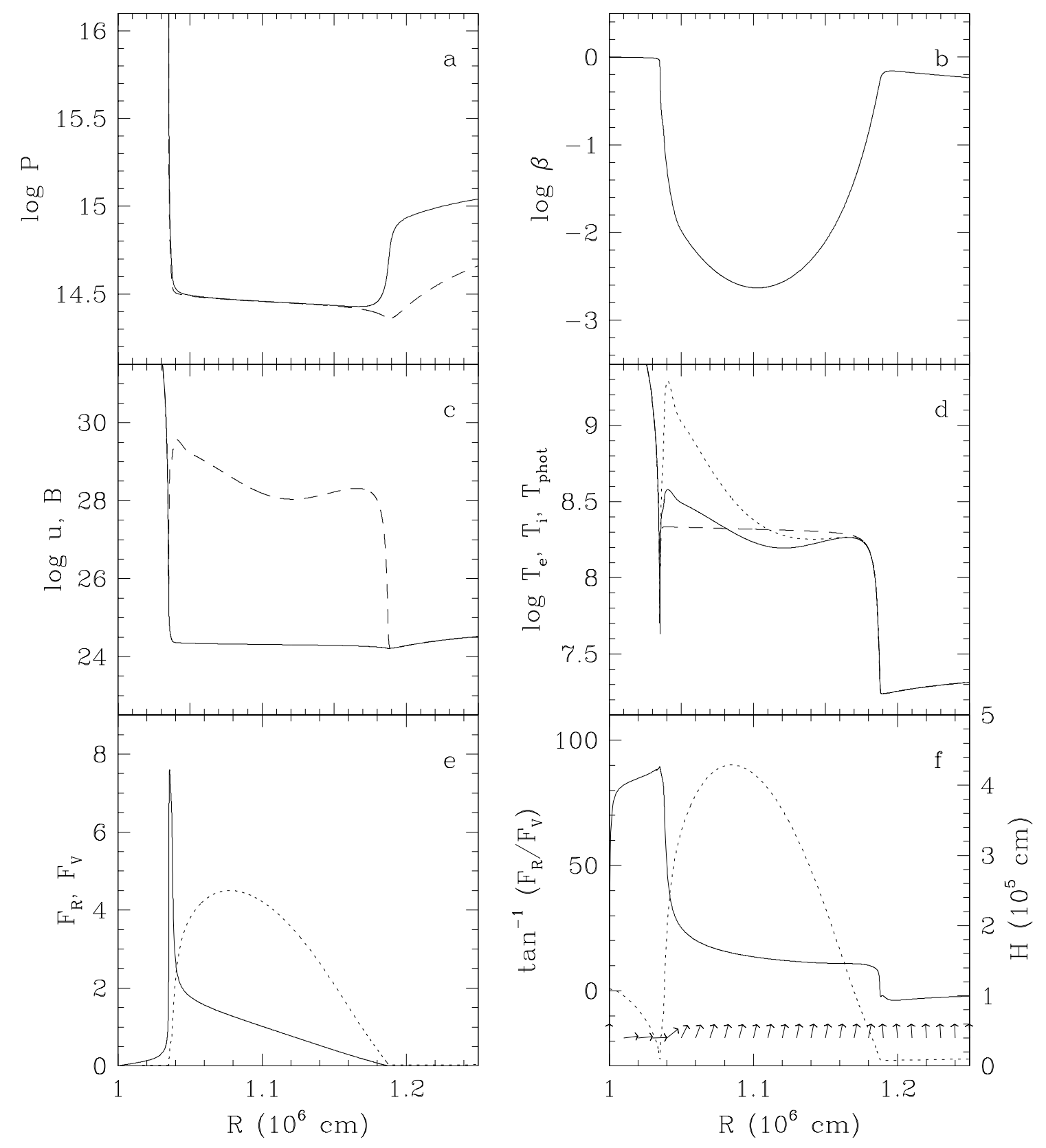

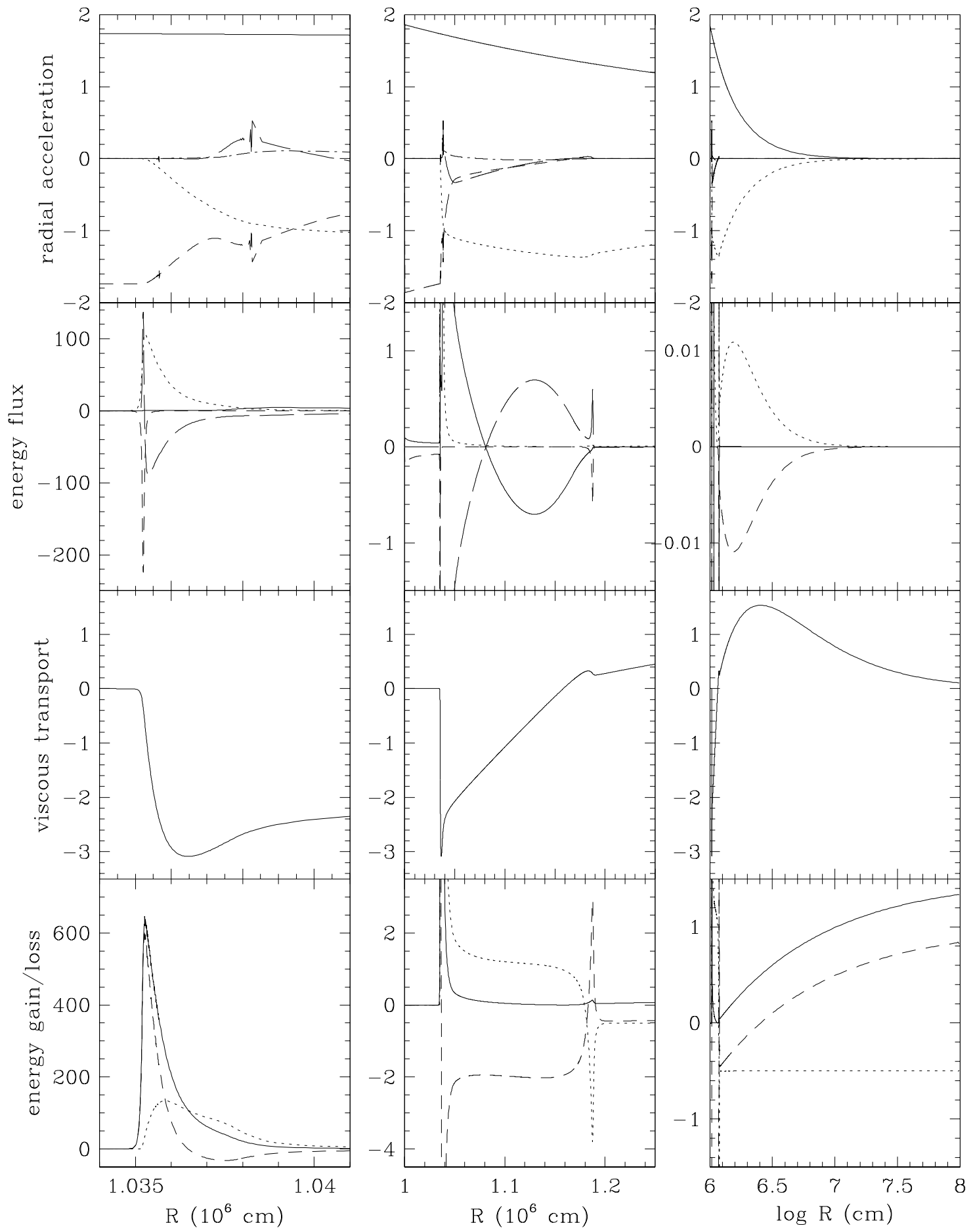

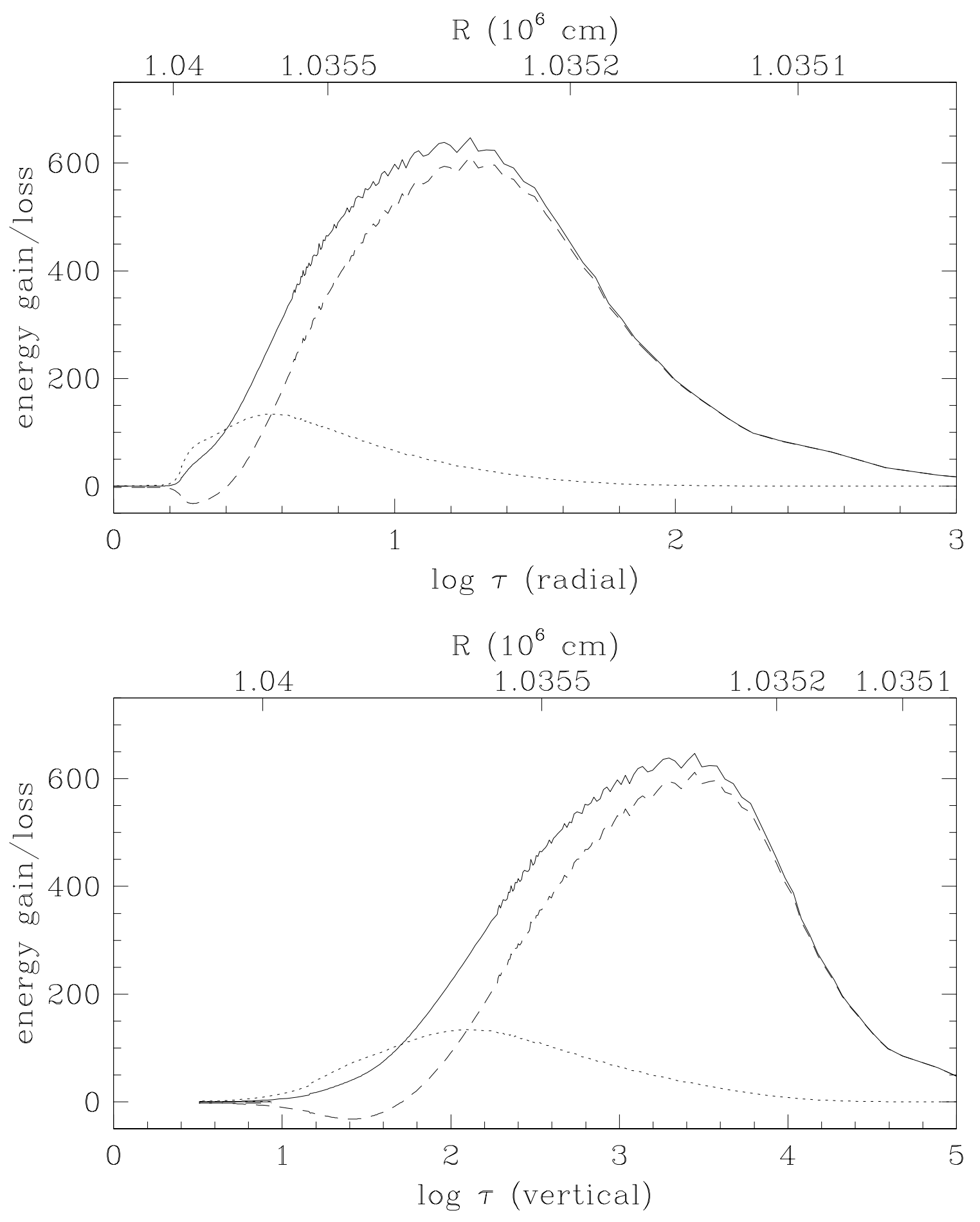

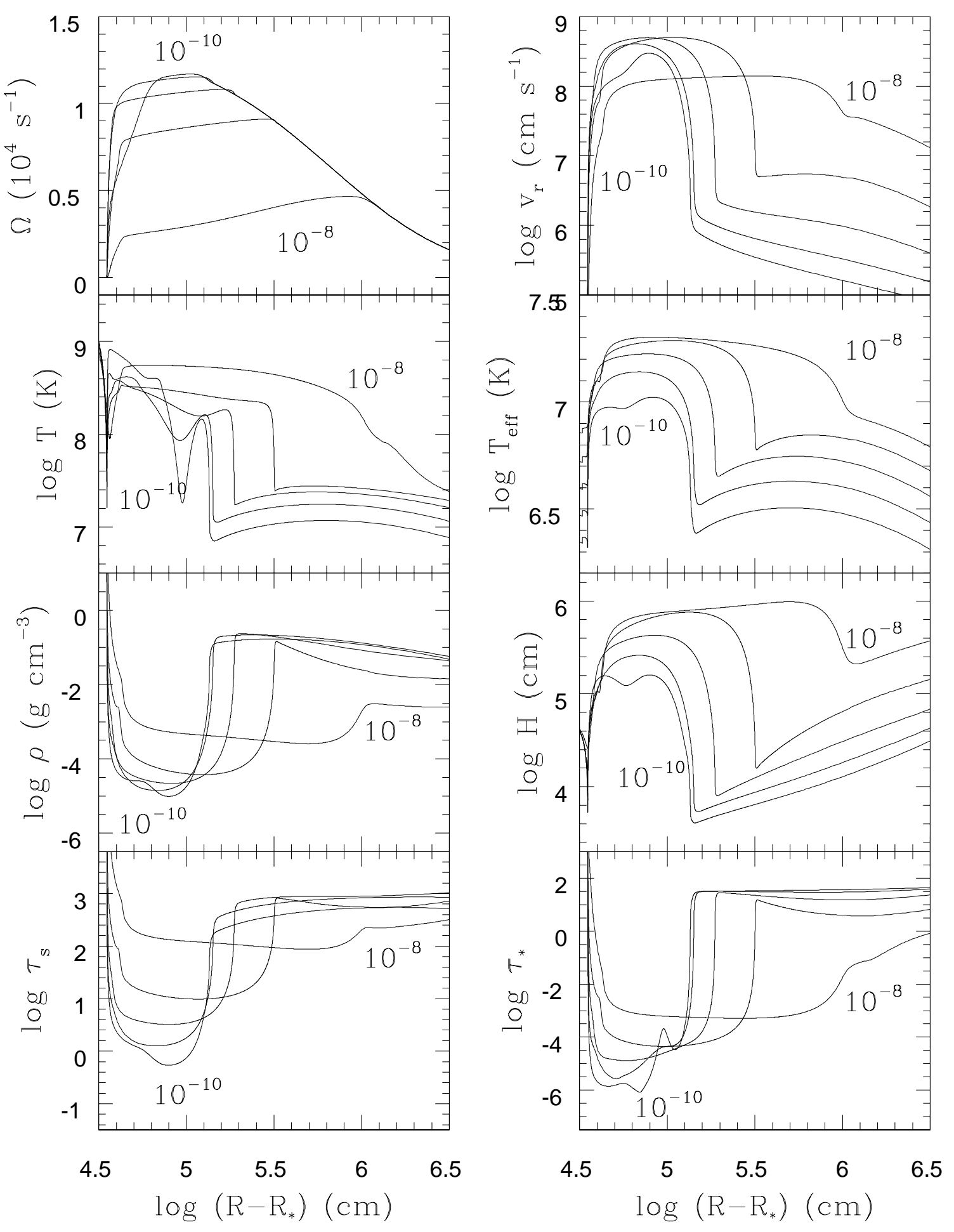


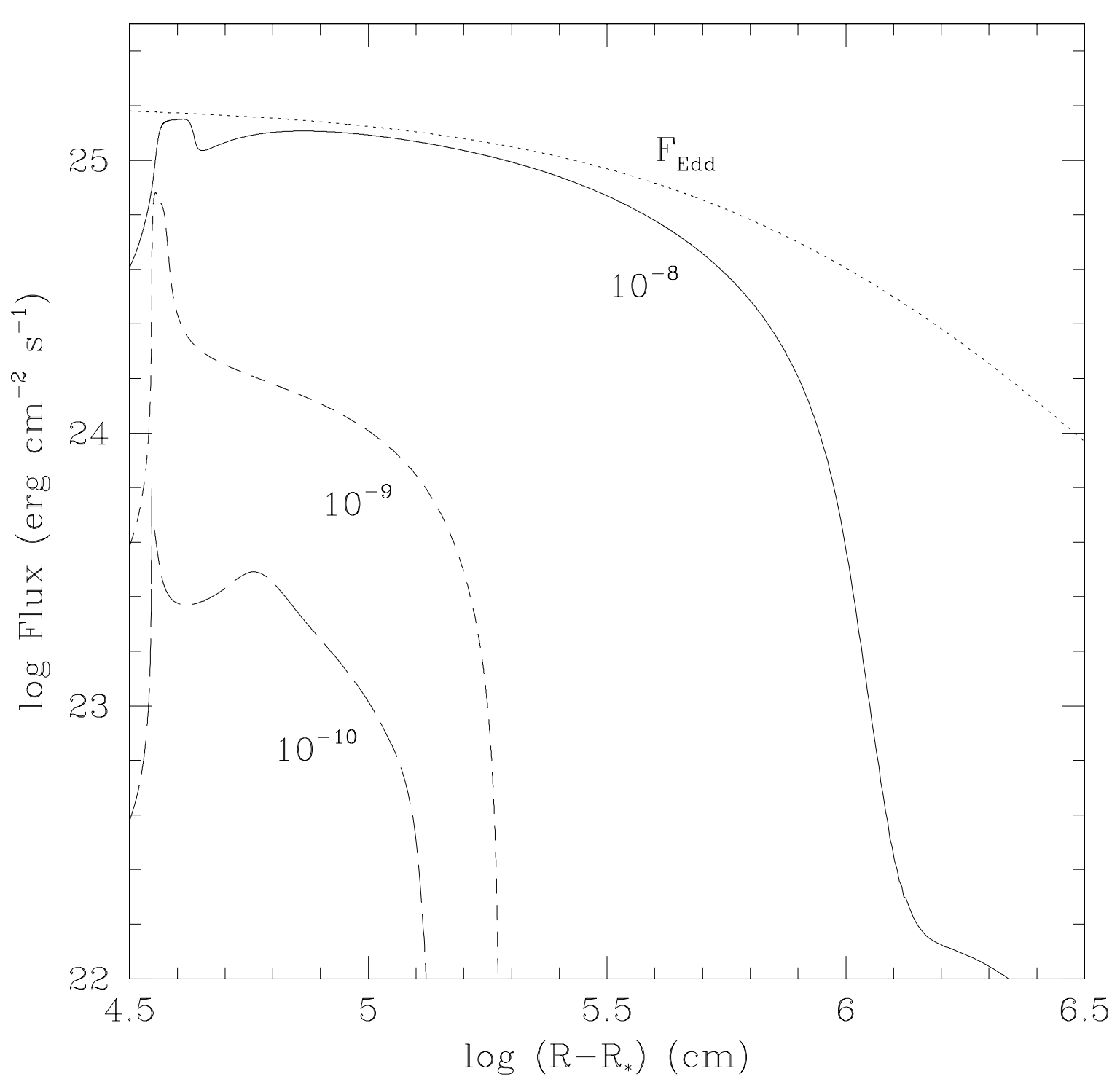



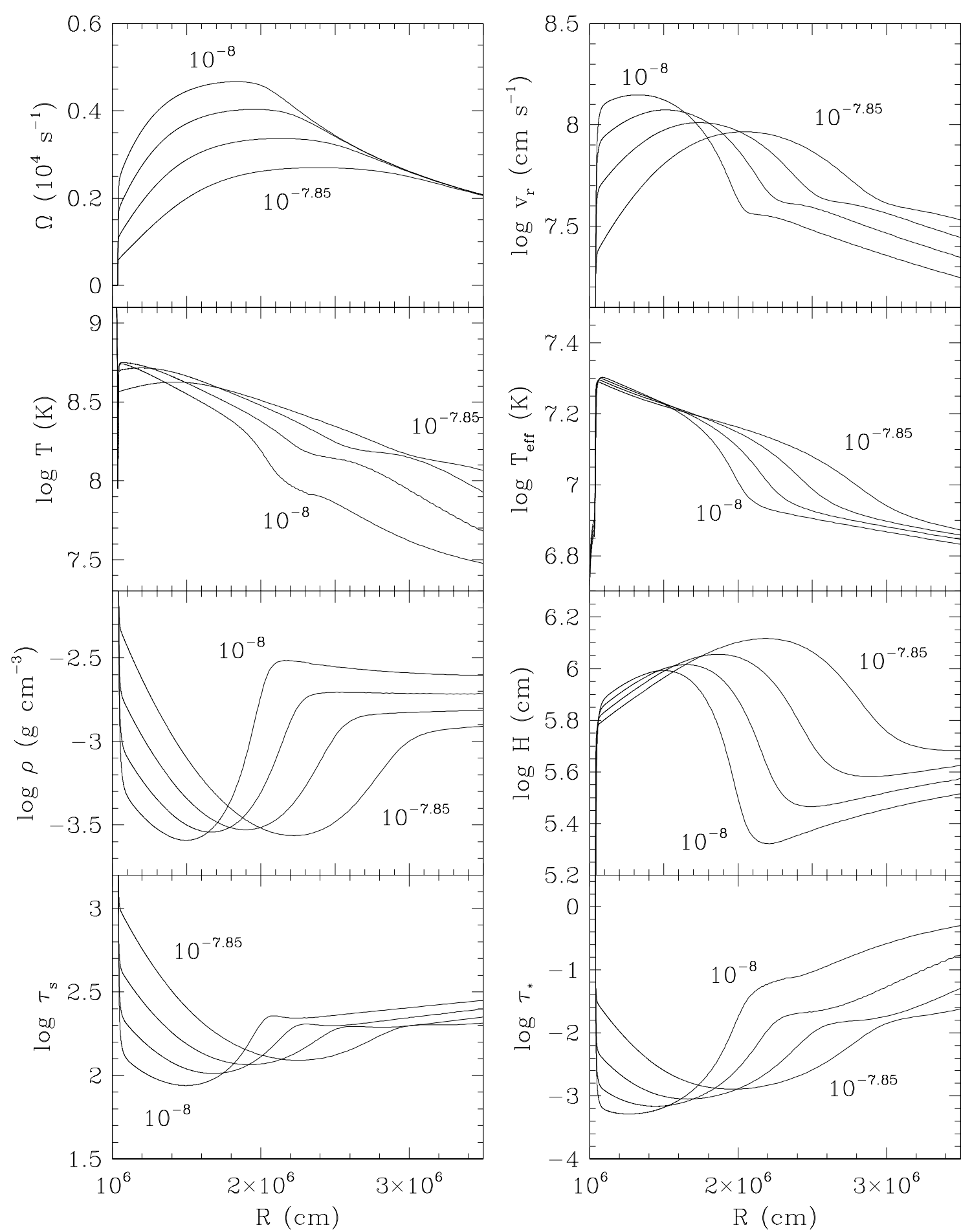

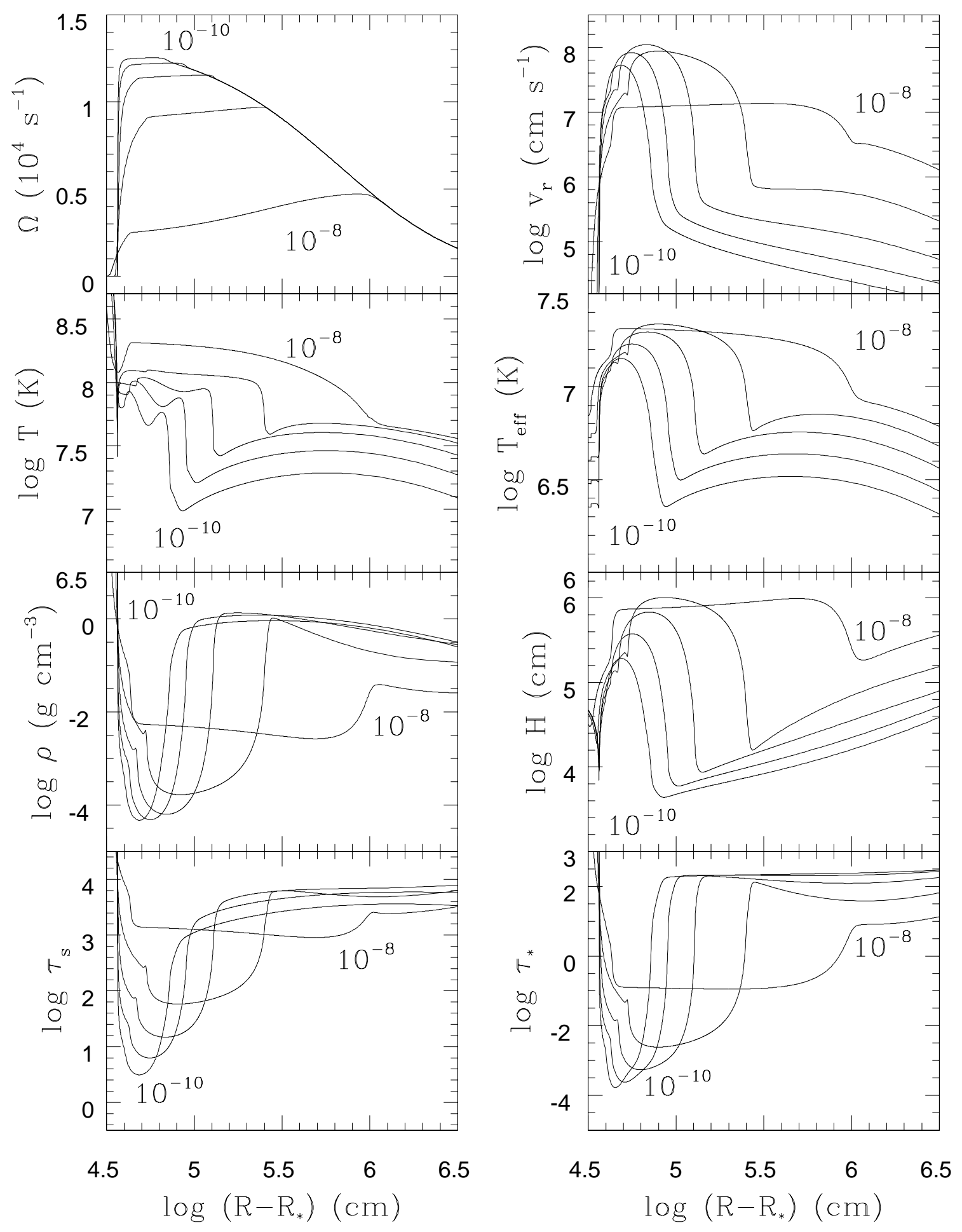

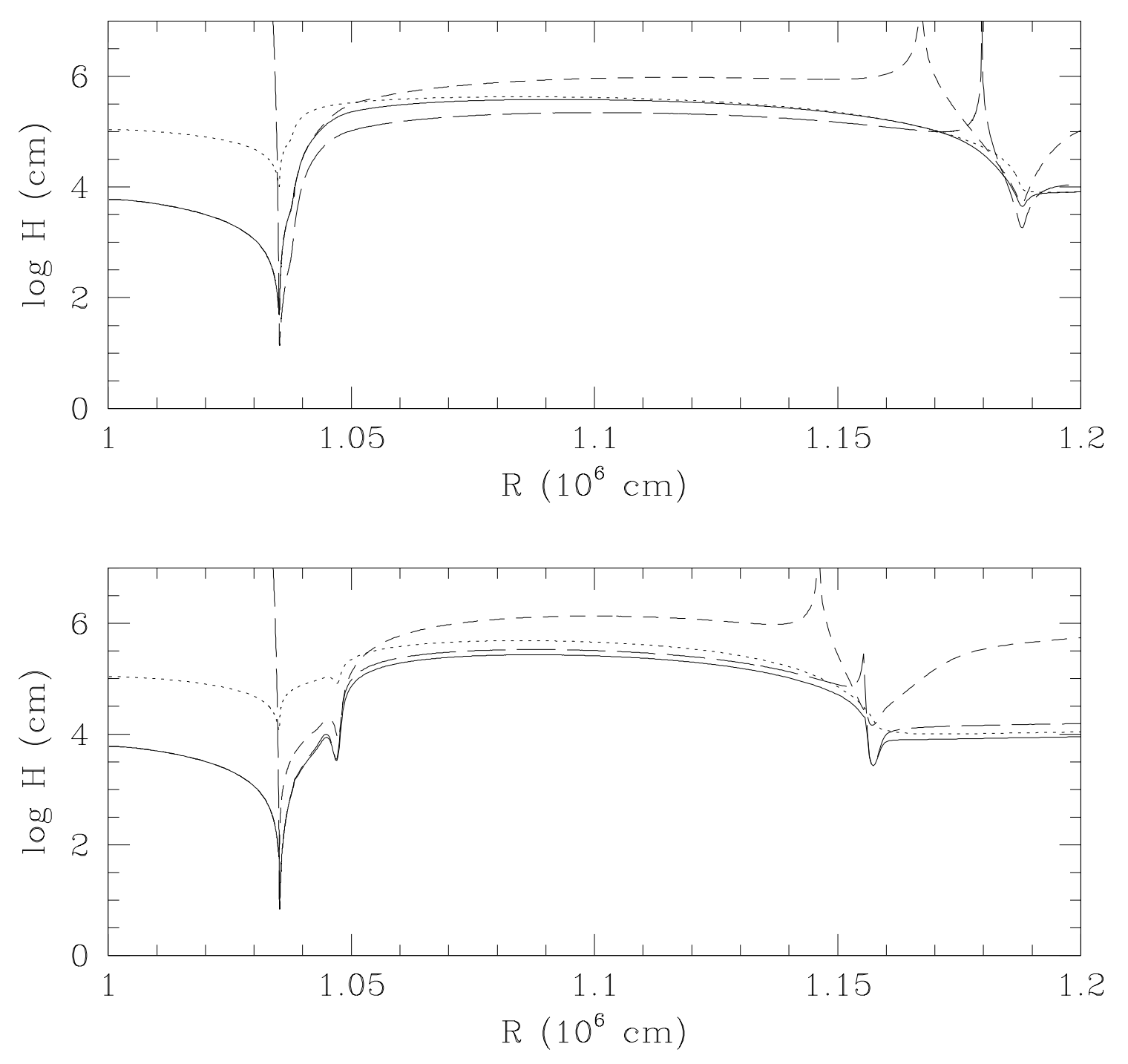

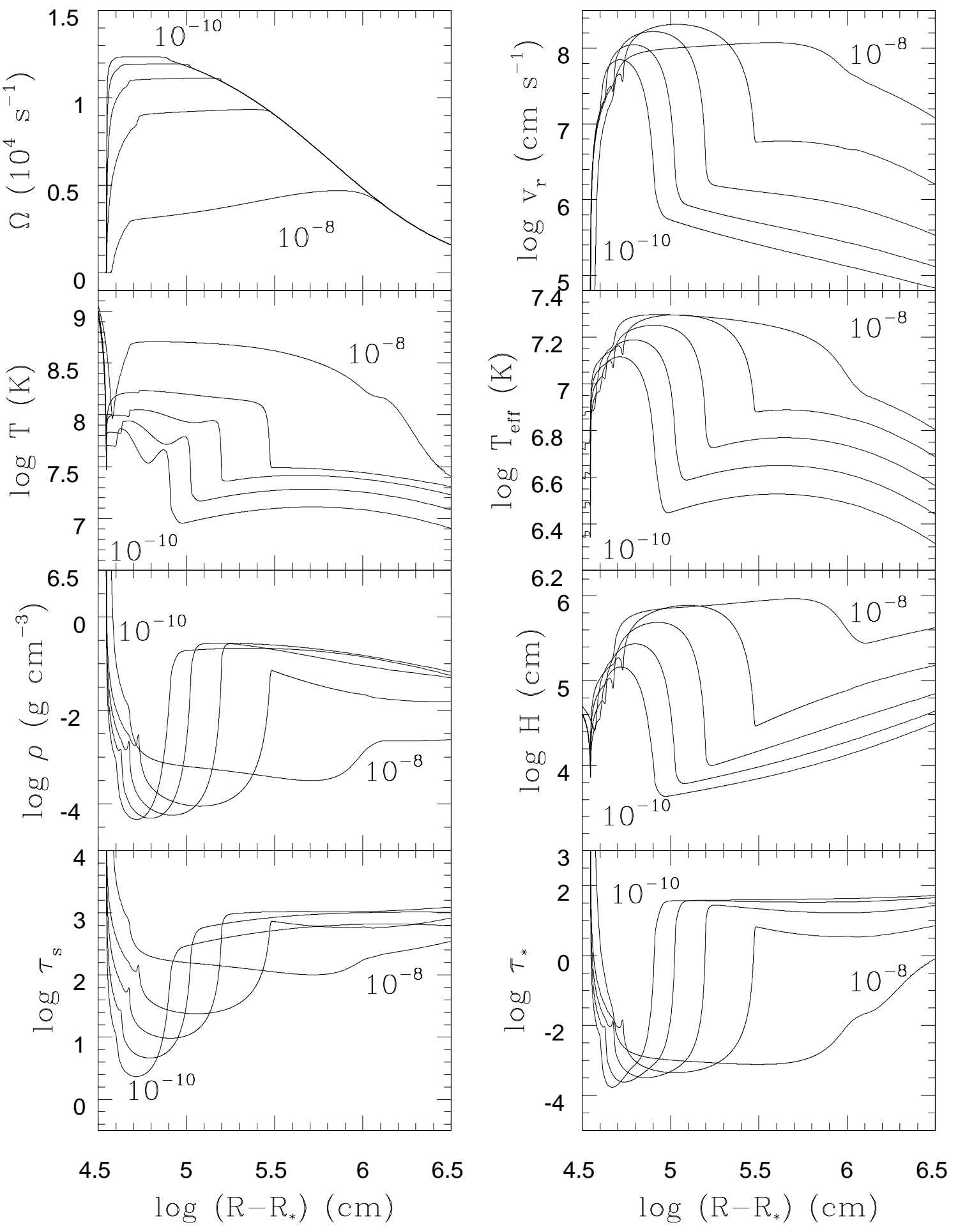

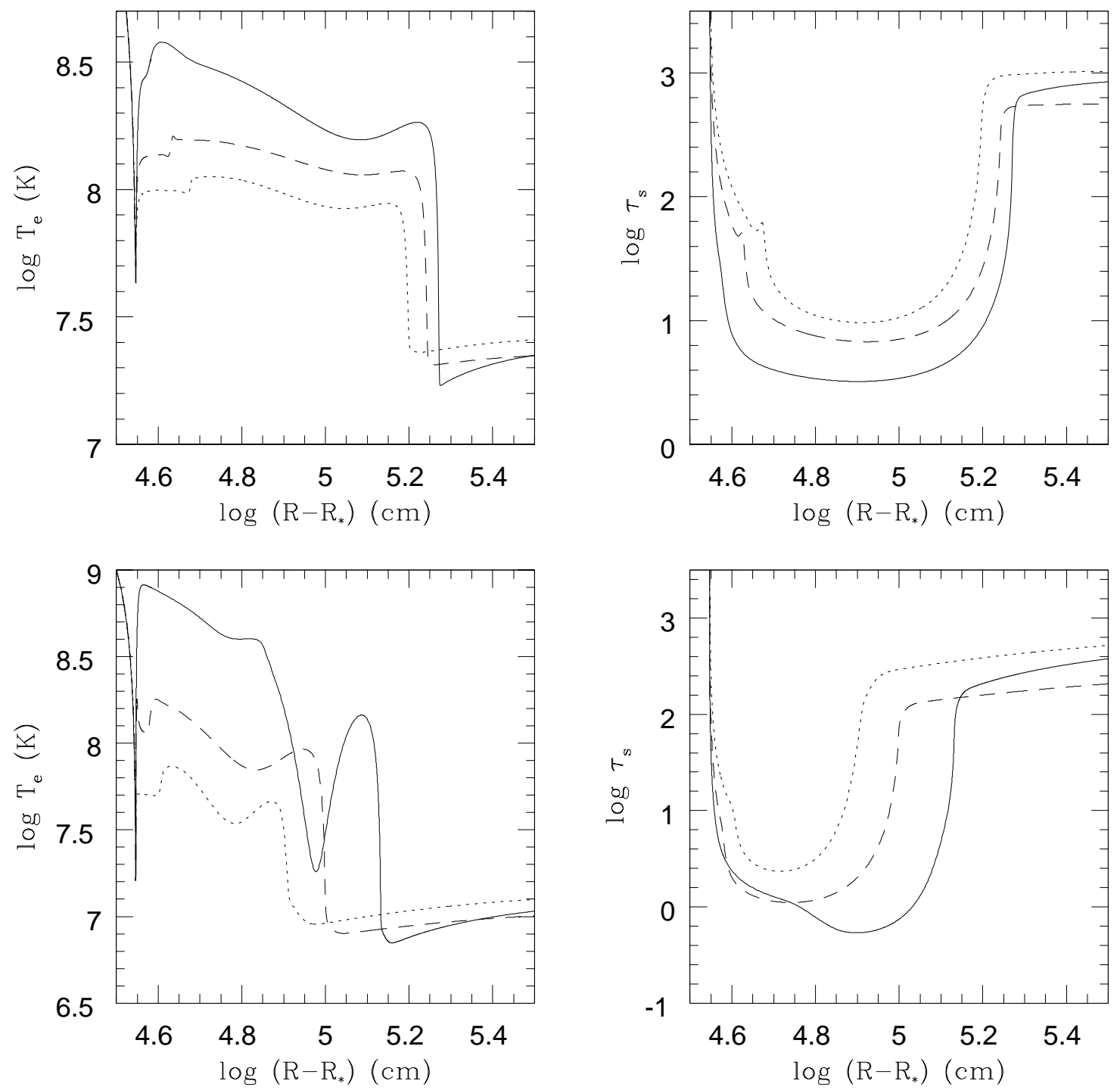

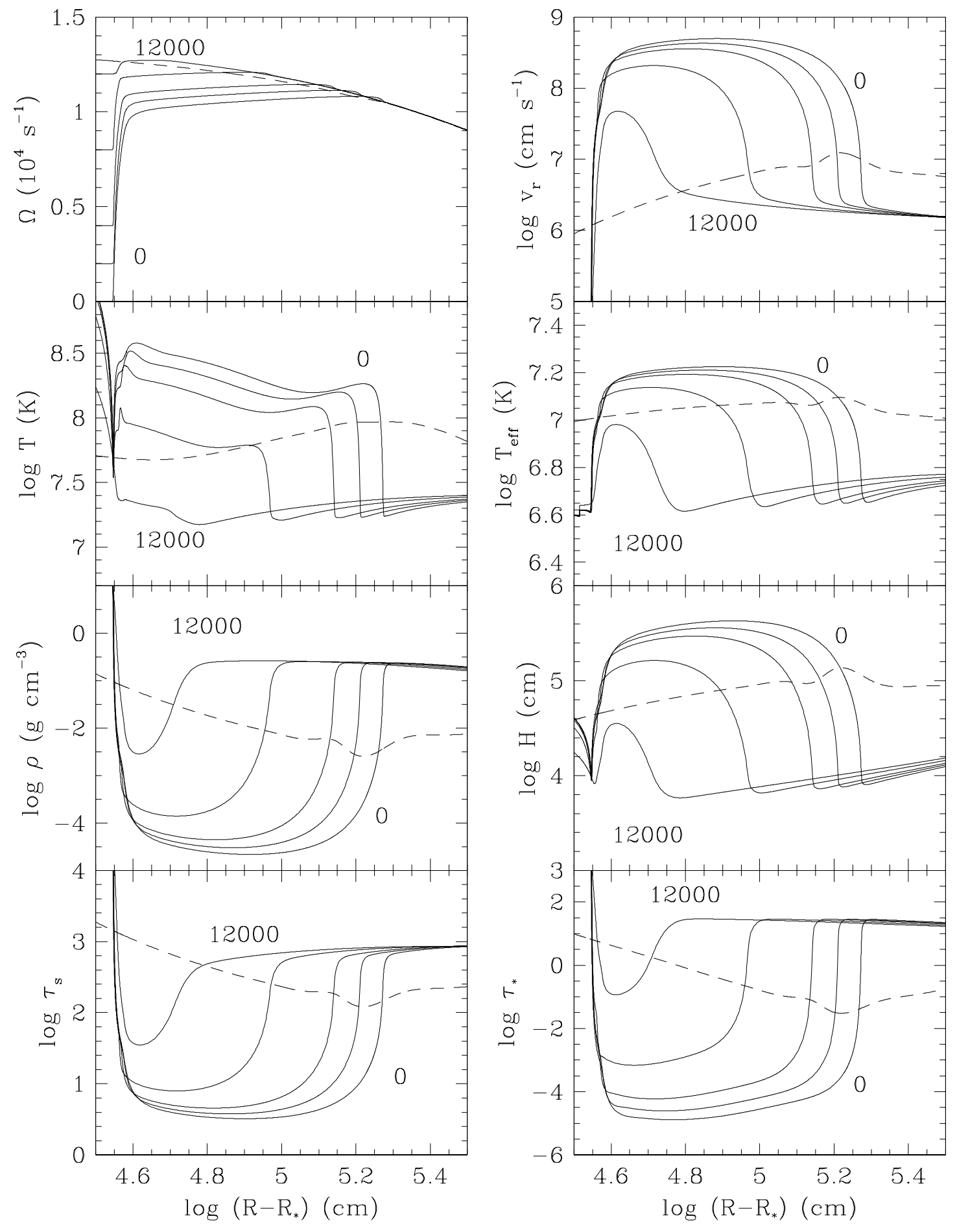